\title{
Solar UV-B and ABA Are Involved in Phenol Metabolism of Vitis vinifera L. Increasing Biosynthesis of Berry Skin Polyphenols
}

\author{
Federico J. Berli, ${ }^{\dagger}$ Martín Fanzone, ${ }^{\dagger}$ Patricia Piccoli, ${ }^{\dagger}$ and Rubén Bottini ${ }^{*,+}$ \\ ${ }^{\dagger}$ Instituto de Biología Agrícola de Mendoza, Facultad de Ciencias Agrarias, CONICET-Universidad Nacional de Cuyo, \\ Almirante Brown 500, M5528AHB, Chacras de Coria, Mendoza, Argentina \\ ${ }^{\ddagger}$ Laboratorio de Aromas y Sustancias Naturales, Estación Experimental Agropecuaria Mendoza, INTA, San Martín 3853, M5507EVY, \\ Mayor Drummond, Mendoza, Argentina
}

\begin{abstract}
It has been previously found that abscisic acid (ABA) participates in the activation of grapevine leaf tissue defense against potentially damaging effects of solar ultraviolet-B radiation (UV-B), apparently by triggering biosynthesis of phenols that filter the harmful radiation and act as antioxidants. The present work studies the effect of solar UV-B and exogenously applied ABA on berry growth, sugar accumulation, and phenol (anthocyanin and nonanthocyanin) profiles across berry development and ripening of Vitis vinifera L. cv. Malbec in a vineyard at $1450 \mathrm{~m}$ of altitude. The grapevines were exposed to relatively high UV-B irradiation (normal sunlight; +UV-B) and also to a reduced UV-B treatment (filter exclusion; - UV-B). These two UV-B treatments were combined with weekly spray applications to the leaves and berries of $1 \mathrm{mM} A B A(+\mathrm{ABA})$ or $\mathrm{H}_{2} \mathrm{O}$ ( $\left.-\mathrm{ABA}\right)$. Reduction of UV-B delayed berry development and maturation, whereas the $+\mathrm{UV}-\mathrm{B}$ and $+\mathrm{ABA}$ combined treatment hastened berry sugar and phenol accumulation. + UV-B/ $+\mathrm{ABA}$ treatments also reduced berry growth and decreased sugar per berry without affecting sugar concentration ( $\left.{ }^{\circ} \mathrm{Brix}\right)$ at harvest. Berry skin $\mathrm{ABA}$ levels were higher in the $+\mathrm{UV}-\mathrm{B}$ and $+\mathrm{ABA}$ combined treatment, which also hastened the onset of ripening up to 20 days. Berry skin $A B A$ levels then decreased toward harvest, implying a possible role for $A B A$ in the control of ripening in this nonclimacteric fruit. Under both + UV-B and $+A B A$ treatments berry skin phenols were additively increased with a change in anthocyanin and nonanthocyanin profiles and increases in the proportion of phenols with high antioxidant capacity.
\end{abstract}

KEYWORDS: UV-B radiation, ABA (abscisic acid), phenolic composition, grapevines, Vitis vinifera L. cv. Malbec

\section{INTRODUCTION}

The levels of solar ultraviolet-B radiation (UV-B; 280$315 \mathrm{~nm}$ ) reaching the earth's surface vary depending on changes in altitude, latitude, season, and time of the day. ${ }^{1}$ UV-B irradiance increases with elevation gain ${ }^{2}$ and comprises about $5 \%$ of the total ultraviolet (UV-B and UV-A; $290-400 \mathrm{~nm}$ ) and $0.5 \%$ of the total solar radiation energy reaching the ground. ${ }^{3}$ Even though relatively low in irradiance, UV-B has enough energy to cause direct and indirect damage to a broad range of cellular constituents, including nucleic acids, lipids, proteins, and cell membranes. ${ }^{4}$ However, plants in nature are seldom visibly damaged by UV-B. Thus, depending on the species, cultivar, and experimental system used, plants can grow and develop under quite diverse UV-B environments, possibly because of effective repair and protection mechanisms. ${ }^{5}$ UV-B radiation has at least two different effects on higher plants. One is the plant's response to the evoked damage, and the other is a response to the perception of UV-B, that is, a response that can best be termed as an induced acclimation. ${ }^{6}$ The plant's responses will depend on the wavelength, fluence rate, and duration of the UV-B irradiation, as well as the length of the adaptation period.

A common protective response against UV-B is the biosynthesis of UV-absorbing compounds that accumulate in epidermal cell vacuoles. ${ }^{7}$ These are mainly phenols that decrease UV penetration into underlying leaf tissues. ${ }^{8}$ UV-B has also been found to increase flavonoids in apple skins ${ }^{9}$ and grape berries. ${ }^{10}$ Some key enzymes involved in the phenylpropanoid (phenylalanine ammonia-lyase, PAL) and flavonoid (chalcone synthase, $\mathrm{CHS}$; and chalcone isomerase, $\mathrm{CHI}$ ) biosynthetic pathways are upregulated by UV-B., ${ }^{41}$

Abscisic acid $(A B A)$ is a phytohormone involved in plant growth and development. It functions by modulating various physiological processes that play key roles in controlling plant responses to biotic and abiotic environmental factors. ${ }^{12}$ In grapevines $\mathrm{ABA}$ participates in the signal transduction and activation of defense mechanisms in UV-B-irradiated leaf tissues by triggering the biosynthesis of different phenols that filter the harmful radiation and/or act as antioxidants. ${ }^{8}$ Thus, berry skin ABA levels increased markedly during the onset of ripening when berries soften and start to accumulate sugars and color develops in red cultivars (veraison). ABA levels then declined to very low levels at ripeness, indicating a possible role of $\mathrm{ABA}$ in initiating veraison. ${ }^{13}$ The assumption that $\mathrm{ABA}$ controls berry maturation in grape, a fruit considered to be nonclimacteric, has also been supported by the finding that ABA applications enhance several processes involved in berry ripening, such as anthocyanin biosynthesis and sugar accumulation. ${ }^{14}$

Phenols are secondary metabolites related to several plant biological functions including seed dispersal, protection against UV radiation, fungal infection, and insect feeding. ${ }^{15}$ Additionally, phenols are important in red wine organoleptic and nutraceutical

Received: January 4, 2011

Revised: $\quad$ April 1, 2011

Accepted: April 6, 2011

Published: April 06, 2011 


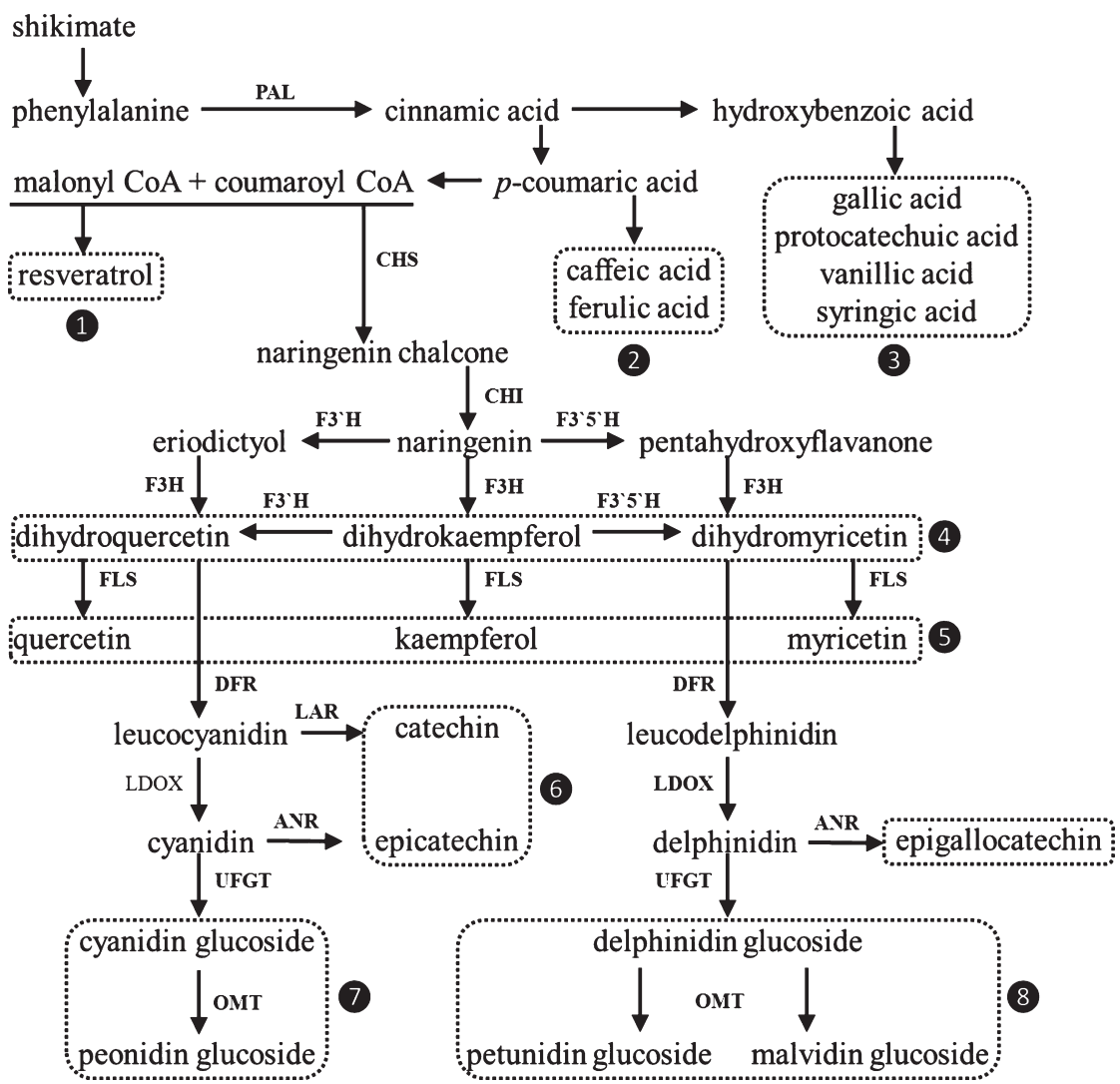

Figure 1. Biosynthetic pathway of the major phenolic compounds of grape (based on ref 35). Subgroups: 1, stilbenes; 2, hydroxycinnamic acids; 3, hydroxybenzoic acids; 4, dihydroflavonols; 5, flavonols; 6, flavanols; 7, dihydroxylated anthocyanins; 8, trihydroxylated anthocyanins. Abbreviations: ANR, anthocyanidin reductase; CHI, chalcone isomerase; CHS, chalcone synthase; DFR, dihydroflavonol reductase; F3H, flavanone 3-hydroxylase; F3'H, flavonoid 3'-hydroxylase; F3' $5^{\prime} \mathrm{H}$, flavonoid $3^{\prime} 5^{\prime}$-hydroxylase; FLS, flavonol synthase; LAR, leucoanthocyanidin reductase; LDOX, leucoanthocyanidin dioxygenase; OMT, o-methyltransferase; PAL, phenylalanine ammonia-lyase; UFGT, flavonoid 3-glucosyltransferase.

characteristics. ${ }^{16}$ Phenols are derived from the phenylpropanoid and flavonoid biosynthetic pathways (Figure 1) and include the phenolic acids (hydroxycinnamic and hydroxybenzoic acids), stilbenes such as resveratrol, and flavonoids (anthocyanins, flavonols, and flavanols). In fact, anthocyanins are the red pigments synthesized in the berry skin at the beginning of veraison. ${ }^{17} \mathrm{Ac}-$ cording to the number and position of hydroxyl and methoxyl groups on the flavan nucleus, they are classified as cyanidin-, peonidin-, delphinidin-, petunidin-, and malvidin-derived anthocyanins. Flavonols are yellow pigments and include quercetin, kaempferol, and myricetin, and they are mainly found as glycosides in the berry skin. ${ }^{18}$ Flavanols are the most abundant class of phenols in grape berries, and they are found as monomers and oligomers, although the majority occur as polymers (tannins) that accumulate mainly before veraison and then decrease toward harvest. $^{19}$

Water restriction is associated with an increased concentration of phenolic compounds, and there is an implicit assumption that water stress augments levels of ABA in the xylem stream. ${ }^{20}$ Thus, high $A B A$ levels and high UV-B irradiation are likely to occur coincidentally in vineyards located at high altitudes. There are no studies that we know of regarding the interaction between $A B A$ and UV-B on the physiology and biochemistry of grape plants, including effects of UV-B on grape berry development and phenolic profile.

The working hypotheses of this experiment were (i) UV-B induces an increase of berry skin $\mathrm{ABA}$ levels, a signal which then enhances the accumulation of phenols as a protective mechanism; (ii) ABA controls the veraison stage of berry development, thereby promoting the onset of ripening; and (iii) exogenously applied ABA can simulate the solar UV-B effects, thereby acting as a signal for the accumulation of phenols.

To test these hypotheses, we applied ABA sprays to grapevines in the absence and presence of UV-B irradiation and then assessed berry skin ABA levels, berry development, berry accumulation of sugar and phenols, and anthocyanin and nonanthocyanin profiles of field-grown Vitis vinifera L. cv. Malbec.

\section{MATERIALS AND METHODS}

Plant Material and Experimental Design. The experiment was carried out during the 2008-2009 season in a commercial vineyard located at high altitude ( $1450 \mathrm{~m}$ above sea level, $69^{\circ} 15^{\prime} 37^{\prime \prime} \mathrm{W}$ and $33^{\circ} 23^{\prime} 51^{\prime \prime} \mathrm{S}$, Gualtallary, Mendoza, Argentina). The grapevines were a selected clone of $V$. vinifera L. cv. Malbec, planted in 1997, own-rooted, trained on a vertical trellis system, and arranged in north-south oriented rows spaced $2 \mathrm{~m}$ apart, with $1.20 \mathrm{~m}$ between plants on the row. The grapevines were pruned to 12 shoots when these shoots reached $10 \mathrm{~cm}$ long, leaving two bunches per shoot. Vines were maintained with no soil-water restriction during the whole experiment by use of a drip irrigation system.

A randomized complete block design with a $2 \times 2$ factorial arrangement of treatments and 5 blocks was used. The experimental unit 
A
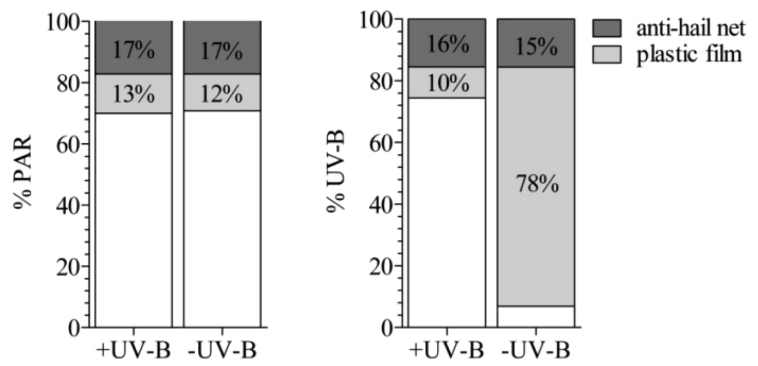

B

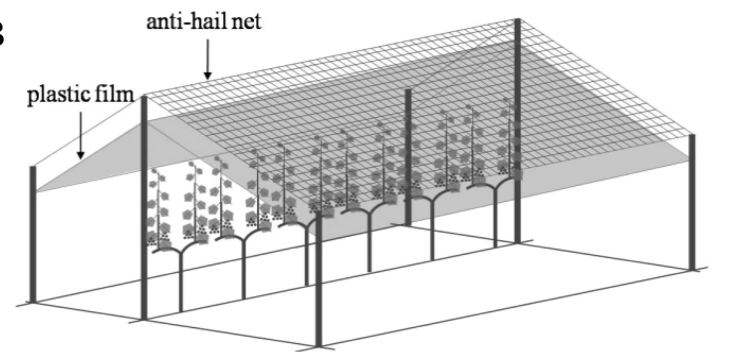

Figure 2. Percentage of solar PAR and UV-B radiation absorbed by the antihail net (dark gray) and by the plastic films (gray) used in +UV-B and -UV-B treatments (PET film and PE filter, respectively), as measured in the field with a quantum sensor and a UV-B detector (A). Schematic representation of an experimental unit (B).

consisted of four plants selected on the basis of their homogeneity from six consecutive plants in the row.

Treatments. Two irradiation regimens were set from 15 days before flowering, stage $23,{ }^{21}$ until harvest (126 days after flowering, DAF). Solar UV-B was filtered with $100 \mu \mathrm{m}$ clear polyester (PE) filters (Oeste Aislante, Buenos Aires, Argentina) to produce a minus UV-B treatment (-UV-B). The PE filter absorbed $78 \%$ of UV-B and $12 \%$ of PAR from the sunlight (Figure 2A). A full UV-B treatment (+UV-B) was set with a $40 \mu \mathrm{m}$ low-density polyethylene (PET) cover to minimize environmental differences between $-\mathrm{UV}-\mathrm{B}$ and $+\mathrm{UV}-\mathrm{B}$. This PET transmitted most of the solar radiation ( $90 \%$ of UV-B and $87 \%$ of photosynthetically active radiation, PAR; Figure $2 \mathrm{~A}$ ). We have previously reported the PE and PET transmittance spectral characteristics. ${ }^{2}$ The plastic sheets were set $2.5 \mathrm{~m}$ above ground level, covering the entire canopy (Figure $2 \mathrm{~B}$ ), and were replaced after breakdown or transmittance reduction, as assessed every 15 days with an LI-250 light meter and an LI-190SA quantum sensor (Licor Inc., Lincoln, NE) and a PMA2200 radiometer with a PMA2102 UV-B detector (Solar Light Co. Inc., Glenside, PA). Both treatments were protected with antihail nets (black polyethylene) that absorbed an extra 15\% of UV-B and 17\% of PAR (Figure 2A).

Hormonal treatments were performed using weekly sprays to the aerial part of the plant (i.e., including leaves and berries) starting 27 days before veraison, stage $35,{ }^{21}$ until harvest. A plus ABA treatment (+ABA) was initiated using a $1 \mathrm{mM}$ aqueous solution of $( \pm)$-S-cis,trans-abscisic acid (90\%, Kelinon Agrochemical Co., Beijing, China). This $(S)$ form of $A B A$ is the naturally occurring higher plant $A B A$. The ABA spray solution also contained $0.1 \%(\mathrm{v} / \mathrm{v})$ of Triton X-100 as emulsificant and a minimum amount of $96 \%$ aqueous ethanol (used to initially dissolve the ABA, ca. $10 \mu \mathrm{L} \mathrm{mg}^{-1}$ of ABA). This dose was chosen according to previous works with grape. ${ }^{8,22}$ The ABA solution was sprayed until runoff (ca. $125 \mathrm{~mL}_{\text {plant }}{ }^{-1}$ ) with a hand-held sprayer and in the late afternoon to minimize $\mathrm{ABA}$ photodegradation. A solution containing $\mathrm{H}_{2} \mathrm{O}$ with the concentration of emulsificant and ethanol described above was used as the control (minus ABA treatment; - ABA).

Berry Sampling and Determinations of Weight, Volume, and Sugar Accumulation. Samples of 55 berries per experimental unit were randomly collected in nylon bags ( 5 berries per bunch, 2 top,
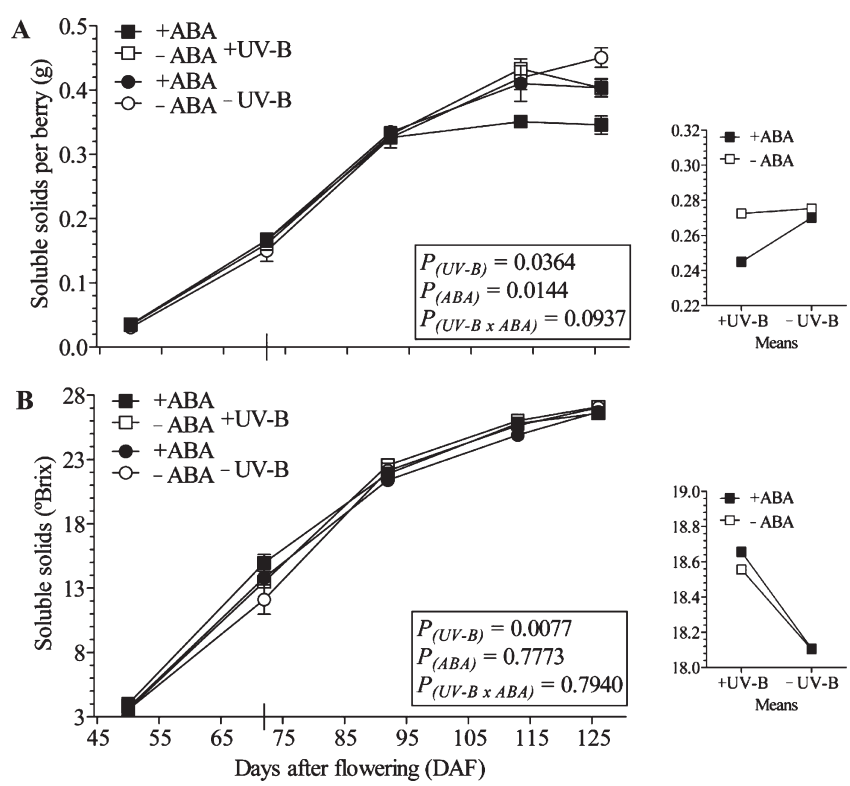

Figure 3. Sugars in grams per berry (A) and ${ }^{\circ}$ Brix (B) at $50,72,92,113$, and $126 \mathrm{DAF}$. +UV-B (squares) and -UV-B (circles) treatments, combined with $+\mathrm{ABA}$ (black) or $-\mathrm{ABA}$ (white) applications. $P_{(\mathrm{UV}-\mathrm{B})}$, UV-B effect; $P_{(\mathrm{ABA})}, \mathrm{ABA}$ effect; $\left.P_{(\mathrm{UV}-\mathrm{B}} \times \mathrm{ABA}\right), \mathrm{UV}-\mathrm{B} \times \mathrm{ABA}$ interaction effect. Values are the mean $\pm S E$, and veraison is indicated with a line at 72 DAF. The figures at the right are the overall means for the treatments.

2 middle, and 1 bottom, from 11 bunches exposed to the west) at 50, 72, 92, 113, and 126 DAF. They were kept in dry ice to prevent dehydration and carried to the laboratory where berry fresh weight (FW) was determined before the berries were frozen and stored at $-20^{\circ} \mathrm{C}$. Then, 50 berries per experimental unit were defrosted at room temperature, and skins were separated from pulps and seeds by hand. The pulps were collected in nylon bags and crushed by hand pressing, and the relative concentration of sugars $\left({ }^{\circ}\right.$ Brix $)$ was measured in the juice with a Pocket PAL-1 digital hand-held refractometer (Atago Co., Ltd., Tokyo, Japan). The ${ }^{\circ}$ Brix was multiplied by the berries FW to calculate sugar on a per berry basis (absolute amounts). The skins obtained as described above were extracted with $50 \mathrm{~mL}$ of an aqueous solution $\left(12 \%\right.$ ethanol, $6 \mathrm{~g} \mathrm{~L}^{-1}$ tartaric acid, $\mathrm{pH} 3.2$ ) at $70{ }^{\circ} \mathrm{C}$ during $3 \mathrm{~h}$ in darkness. Then the liquid fraction was separated by decanting, maintained for $24 \mathrm{~h}$ at $4{ }^{\circ} \mathrm{C}$, and centrifuged for $10 \mathrm{~min}$ at $10000 \mathrm{~g}$ to eliminate tartrates and other sediments. The supernatant was collected, frozen, and stored at $-20^{\circ} \mathrm{C}$.

The other five berries per experimental unit were defrosted at room temperature and used to determine berry volume (water displacement) and dry weight (DW) of the skins, pulps, and seeds (dried at $40^{\circ} \mathrm{C}$ until constant weight).

Abscisic Acid in Berry Skin. One hundred nanograms of $\left({ }^{2} \mathrm{H}_{6}\right)$ ABA (a gift from Professor J. D. Cohen, University of Minnesota) dissolved in $10 \mu \mathrm{L}$ of methanol was added to an aliquot of $0.5 \mathrm{~mL}$ of berry skin extraction solution and the isotopes allowed to equilibrate for $1 \mathrm{~h}$ at $4{ }^{\circ} \mathrm{C}$. Then, $2 \mathrm{~mL}$ of $80 \%$ aqueous methanol in $1 \%$ acetic acid was added, and the sample was evaporated in a rotavapor with vacuum at $35{ }^{\circ} \mathrm{C}$. Further purification and derivatization were performed prior to quantification of ABA by capillary gas chromatography-electron impact mass spectrometry (GC-EIMS) with Clarus 500 (PerkinElmer Shelton, $\mathrm{CT}$ ) equipment as described in ref 8.

Anthocyanins and Nonanthocyanin Phenols in Berry Skin. For anthocyanins, aliquots of $150 \mu \mathrm{L}$ of berry skin extraction solution were filtered through a $0.45 \mu \mathrm{m}$ pore size nylon membrane, and then $100 \mu \mathrm{L}$ was injected in high-performance liquid chromatography equipment with a photodiode array detector (HPLC-DAD; series 200, 
Table 1. Berry Volume, Berry FW, Berry DW, Skin DW, Pulp DW, Seeds DW, and Skin DW to Berry DW at 126 DAF

\begin{tabular}{|c|c|c|c|c|c|c|c|c|}
\hline \multicolumn{2}{|c|}{ treatment } & $\begin{array}{l}\text { berry volume } \\
\qquad(\mathrm{mL})\end{array}$ & $\begin{array}{l}\text { berry FW } \\
(\mathrm{mg})\end{array}$ & $\begin{array}{l}\text { berry DW } \\
(\mathrm{mg})\end{array}$ & $\begin{array}{c}\text { skin DW } \\
\text { (mg per berry) }\end{array}$ & $\begin{array}{c}\text { pulp DW } \\
\text { (mg per berry) }\end{array}$ & $\begin{array}{c}\text { seeds DW } \\
\text { (mg per berry) }\end{array}$ & $\begin{array}{c}\text { skin DW to } \\
\text { berry DW (\%) }\end{array}$ \\
\hline \multirow[t]{2}{*}{+ UV-B } & $+\mathrm{ABA}$ & $1.1 \pm 0.0$ & $1298.5 \pm 43.4$ & $377.3 \pm 24.1$ & $105.9 \pm 7.2$ & $230.1 \pm 12.9$ & $41.2 \pm 4.8$ & $28.1 \pm 0.3$ \\
\hline & $-\mathrm{ABA}$ & $1.3 \pm 0.1$ & $1488.4 \pm 50.1$ & $442.3 \pm 25.7$ & $120.7 \pm 6.6$ & $276.4 \pm 14.9$ & $45.3 \pm 5.0$ & $27.3 \pm 0.5$ \\
\hline \multirow[t]{2}{*}{$-\mathrm{UV}-\mathrm{B}$} & $+\mathrm{ABA}$ & $1.4 \pm 0.1$ & $1514.5 \pm 69.3$ & $456.1 \pm 18.9$ & $132.1 \pm 3.8$ & $268.0 \pm 14.3$ & $55.9 \pm 3.4$ & $29.1 \pm 1.1$ \\
\hline & $-\mathrm{ABA}$ & $1.6 \pm 0.1$ & $1667.3 \pm 62.3$ & $546.5 \pm 33.3$ & $150.0 \pm 6.1$ & $335.5 \pm 23.9$ & $61.0 \pm 5.7$ & $27.6 \pm 0.9$ \\
\hline \multicolumn{2}{|l|}{$P_{\text {(UV-B) }}$} & 0.0010 & 0.0106 & 0.0049 & 0.0008 & 0.0188 & 0.0035 & 0.4263 \\
\hline \multicolumn{2}{|c|}{$P_{(\mathrm{ABA})}$} & 0.0191 & 0.0222 & 0.0127 & 0.0224 & 0.0079 & 0.2951 & 0.2018 \\
\hline \multicolumn{2}{|c|}{$P_{(\mathrm{UV}-\mathrm{B} \times \mathrm{ABA})}$} & 1.0000 & 0.7811 & 0.6415 & 0.8013 & 0.5641 & 0.9082 & 0.6538 \\
\hline
\end{tabular}

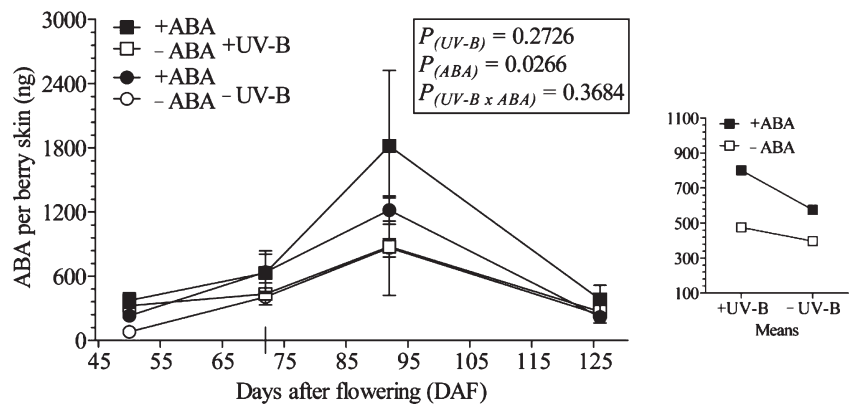

Figure 4. ABA (ng per berry skin) at 50, 72, 92, and 126 DAF: +UV-B (squares) and - UV-B (circles) treatments, combined with $+\mathrm{ABA}$ (black) or $-\mathrm{ABA}$ (white) applications. $P_{(\mathrm{UV}-\mathrm{B})}, \mathrm{UV}-\mathrm{B}$ effect; $P_{(\mathrm{ABA})}$,

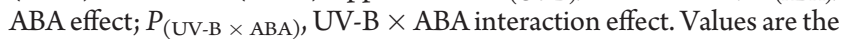
mean $\pm S E$, and veraison is indicated with a line at $72 \mathrm{DAF}$. The figure at the right is the overall mean for the treatments.

PerkinElmer). Separation was performed on a reverse-phase Chromolith Performance $\mathrm{C}_{18}$ column (100 mm length, $4.6 \mathrm{~mm}$ inner diameter, and $2 \mu \mathrm{m}$ film thickness; Merck, Darmstadt, Germany), eluted at $25^{\circ} \mathrm{C}$ with a gradient of solvent $\mathrm{A}\left(10 \%\right.$ formic acid in $\left.\mathrm{H}_{2} \mathrm{O}\right)$ and solvent $\mathrm{B}$ ( $100 \%$ acetonitrile) applied at a flow rate of $1.1 \mathrm{~mL} \mathrm{~min}^{-1}$ from 0 to 22 $\mathrm{min}$ and of $1.5 \mathrm{~mL} \mathrm{~min}^{-1}$ from 22 to $35 \mathrm{~min}$ as follows: $4-15 \% \mathrm{~B}$ from 0 to $12 \mathrm{~min}, 15 \% \mathrm{~B}$ from 12 to $22 \mathrm{~min}, 15-30 \%$ B from 22 to $35 \mathrm{~min}$, followed by a final wash with $100 \%$ methanol. Detection was performed from 210 to $600 \mathrm{~nm}$, according to ref 23, and the quantification was carried out by peak area calculation at $520 \mathrm{~nm}$. Anthocyanin amount was expressed by using malvidin 3-glucoside (Extrasynthese, Lyon, France) as standard for a calibration curve $\left(r^{2}=0.96\right)$.

For nonanthocyanin phenols, aliquots of $10 \mathrm{~mL}$ of berry skin extraction solution were added together with $1 \mathrm{~g}$ of $\mathrm{NaCl}$ and subjected to three successive extractions with $5 \mathrm{~mL}$ of diethyl ether and $5 \mathrm{~mL}$ of ethyl acetate. The organic fractions were combined, dehydrated with $2.5 \mathrm{~g}$ of $\mathrm{Na}_{2} \mathrm{SO}_{4}$, filtered through a $3 \mu \mathrm{m}$ pore size cellulose filter, and evaporated to dryness under a gentle $\mathrm{N}_{2}$ gas stream at $30^{\circ} \mathrm{C}$. The solid residue was diluted with $0.4 \mathrm{~mL}$ of $50 \%$ methanol in $\mathrm{H}_{2} \mathrm{O}$ and filtered through a $0.45 \mu \mathrm{m}$ pore size nylon membrane, and then $30 \mu \mathrm{L}$ was injected in the HPLC-DAD equipment with a reverse-phase Nova-Pak $\mathrm{C}_{18}$ column (300 mm length, $3.9 \mathrm{~mm}$ inner diameter, and $4 \mu \mathrm{m}$ film thickness; Waters Corp., Milford, MA). A gradient consisting of solvent $\mathrm{C}\left(2 \%\right.$ acetic acid in $\left.\mathrm{H}_{2} \mathrm{O}\right)$ and solvent $\mathrm{D}\left(\mathrm{H}_{2} \mathrm{O}\right.$ /acetonitrile/acetic acid, 78:20:2) was applied at a flow rate of $0.9 \mathrm{~mL} \mathrm{~min}^{-1}$ from 0 to $55 \mathrm{~min}$ and of $1.0 \mathrm{~mL} \mathrm{~min}^{-1}$ from 55 to 125 min as follows: $0-80 \% \mathrm{D}$ from 0 to $55 \mathrm{~min}, 80-90 \% \mathrm{D}$ from 55 to $57 \mathrm{~min}$, $90 \% \mathrm{D}$ from 57 to $70 \mathrm{~min}, 90-100 \% \mathrm{D}$ from 70 to $80 \mathrm{~min}, 100 \% \mathrm{D}$ from 80 to $125 \mathrm{~min}$, followed by a $100 \%$ methanol washing. Detection was

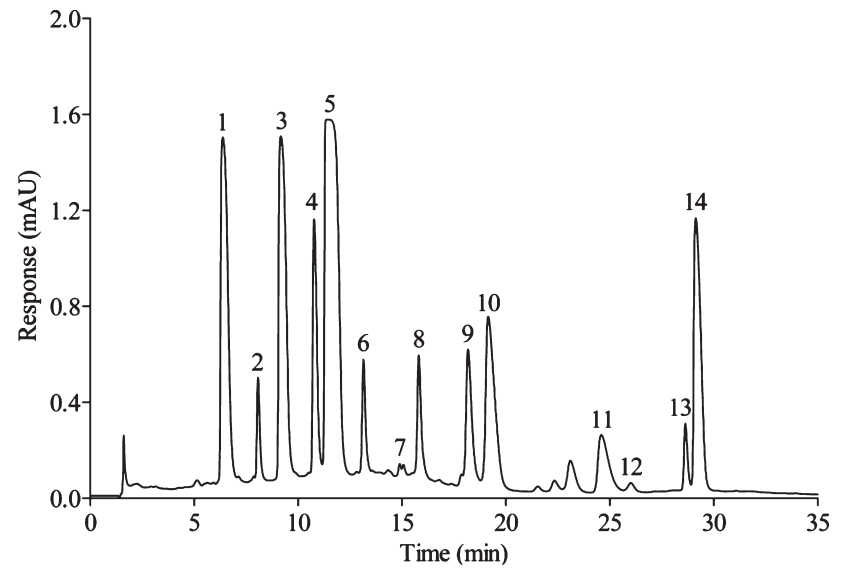

Figure 5. Typical HPLC-DAD chromatogram of berry skin anthocyanins. Nonacylated glucosides: delphinidin (1); cyanidin (2); petunidin (3); peonidin (4); and malvidin (5). Acetyl glucosides: delphinidin (6); cyanidin (7); petunidin (8); peonidin (9); and malvidin (10). p-Coumaroyl glucosides: delphinidin (11); petunidin (12); peonidin (13); and malvidin (14).

performed from 210 to $360 \mathrm{~nm}$, and the identification was done by comparison of $\mathrm{UV}$ - vis spectra and retention times with those of standards purchased from Sigma Chemical Co. (St. Louis, MO): gallic, protocatechuic, vanillic, syringic, $p$-coumaric, and caffeic acids; tyrosol, (+)-catechin, (-)-epicatechin, trans-resveratrol, myricetin, quercetin, quercetin 3-galactoside, and quercetin 3-glucoside. The compounds for which no standards were available were identified by their retention time and UV-vis spectral characteristics. Quantification was performed with the calibration curves obtained by injection of standard solutions under the same conditions as for the samples analyzed, over the range of concentrations observed $\left(r^{2} \geq 0.94\right)$. The compounds without standards were quantified with the curves of chemically related compounds, that is, quercetin for dihydroflavonols; quercetin 3-glucoside for quercetin 3-rhamnoside, myricetin glycosides, and kaempferol glycosides; caffeic acid for caftaric acid; $p$-coumaric acid for coutaric acid; and (+)-catechin for procyanidins.

Statistical Analysis. Statistical analyses were performed with the software Statgraphics Centurion XV version 15.0.10 (Statpoint Technologies Inc., Warrenton, VA). The effects of UV-B, ABA, and their interaction were determinated by multifactorial ANOVA.

\section{RESULTS}

Berry FW and sugar accumulation increased from 50 to 126 DAF following a typical sigmoid curve, where veraison occurred 
Table 2. Total Anthocyanin Glucosides (Micrograms per Berry Skin) and Relative Abundance of Trihydroxylated and Methoxylated Anthocyanins at 50, 72, 92, 113, and $126 \mathrm{DAF}^{a}$

\begin{tabular}{|c|c|c|c|c|c|c|c|c|}
\hline \multirow[b]{2}{*}{ compound } & \multirow[b]{2}{*}{ DAF } & \multicolumn{2}{|c|}{+ UV-B } & \multicolumn{2}{|c|}{$-U V-B$} & \multirow[b]{2}{*}{$P_{(\text {UV-B })}$} & \multirow[b]{2}{*}{$P_{(\mathrm{ABA})}$} & \multirow[b]{2}{*}{$\left.P_{(\mathrm{UV}-\mathrm{B}} \times \mathrm{ABA}\right)$} \\
\hline & & $+\mathrm{ABA}$ & $-\mathrm{ABA}$ & $+\mathrm{ABA}$ & $-\mathrm{ABA}$ & & & \\
\hline \multirow[t]{5}{*}{ nonacylated } & 50 & $1.2 \pm 0.3$ & $0.6 \pm 0.3$ & $1.2 \pm 0.2$ & $0.7 \pm 0.2$ & 0.0003 & 0.0105 & 0.6934 \\
\hline & 72 & $353.6 \pm 15.4$ & $185.0 \pm 31.3$ & $265.7 \pm 72.0$ & $183.2 \pm 41.4$ & & & \\
\hline & 92 & $935.4 \pm 128.6$ & $717.4 \pm 32.6$ & $746.9 \pm 65.0$ & $682.9 \pm 30.5$ & & & \\
\hline & 113 & $875.3 \pm 61.5$ & $916.6 \pm 78.6$ & $747.4 \pm 78.4$ & $722.9 \pm 60.8$ & & & \\
\hline & 126 & $965.0 \pm 48.9$ & $924.1 \pm 15.2$ & $829.7 \pm 113.7$ & $714.9 \pm 72.7$ & & & \\
\hline \multirow[t]{5}{*}{ acetylated } & 50 & $0.0 \pm 0.0$ & $0.0 \pm 0.0$ & $0.0 \pm 0.0$ & $0.0 \pm 0.0$ & 0.0081 & 0.1561 & 0.9530 \\
\hline & 72 & $72.2 \pm 4.7$ & $38.2 \pm 7.9$ & $61.7 \pm 16.8$ & $37.1 \pm 7.1$ & & & \\
\hline & 92 & $299.0 \pm 31.6$ & $231.3 \pm 8.2$ & $241.3 \pm 26.2$ & $221.7 \pm 13.5$ & & & \\
\hline & 113 & $267.1 \pm 31.5$ & $302.2 \pm 30.1$ & $243.5 \pm 37.8$ & $235.3 \pm 6.6$ & & & \\
\hline & 126 & $303.1 \pm 9.9$ & $297.4 \pm 17.2$ & $259.0 \pm 52.4$ & $244.9 \pm 25.7$ & & & \\
\hline \multirow[t]{5}{*}{$p$-coumaroylated } & 50 & $0.1 \pm 0.0$ & $0.0 \pm 0.0$ & $0.1 \pm 0.0$ & $0.1 \pm 0.0$ & 0.0148 & 0.6080 & 0.9540 \\
\hline & 72 & $40.4 \pm 4.4$ & $19.0 \pm 3.2$ & $29.8 \pm 9.5$ & $16.6 \pm 3.1$ & & & \\
\hline & 92 & $179.4 \pm 16.1$ & $132.4 \pm 1.4$ & $136.0 \pm 13.7$ & $135.1 \pm 9.7$ & & & \\
\hline & 113 & $182.6 \pm 27.5$ & $217.2 \pm 22.6$ & $168.7 \pm 27.7$ & $165.0 \pm 2.1$ & & & \\
\hline & 126 & $212.1 \pm 30.7$ & $224.1 \pm 24.0$ & $181.0 \pm 33.9$ & $181.5 \pm 22.5$ & & & \\
\hline \multirow[t]{5}{*}{ delphinidins } & 50 & $0.2 \pm 0.1$ & $0.1 \pm 0.1$ & $0.3 \pm 0.1$ & $0.1 \pm 0.0$ & 0.0004 & 0.0359 & 0.8505 \\
\hline & 72 & $90.8 \pm 5.0$ & $44.1 \pm 8.6$ & $66.2 \pm 19.1$ & $43.5 \pm 10.2$ & & & \\
\hline & 92 & $303.0 \pm 54.0$ & $224.3 \pm 12.1$ & $237.3 \pm 26.4$ & $204.0 \pm 15.0$ & & & \\
\hline & 113 & $261.9 \pm 22.6$ & $284.8 \pm 35.8$ & $209.9 \pm 26.9$ & $203.3 \pm 28.0$ & & & \\
\hline & 126 & $287.1 \pm 19.9$ & $290.5 \pm 9.8$ & $245.7 \pm 48.7$ & $190.1 \pm 28.9$ & & & \\
\hline \multirow[t]{5}{*}{ cyanidins } & 50 & $0.0 \pm 0.0$ & $0.0 \pm 0.0$ & $0.0 \pm 0.0$ & $0.0 \pm 0.0$ & 0.0001 & 0.0052 & 0.3736 \\
\hline & 72 & $19.2 \pm 3.5$ & $9.1 \pm 2.0$ & $13.2 \pm 3.2$ & $8.5 \pm 2.3$ & & & \\
\hline & 92 & $55.2 \pm 17.8$ & $33.1 \pm 0.9$ & $29.1 \pm 4.4$ & $23.8 \pm 3.4$ & & & \\
\hline & 113 & $46.5 \pm 11.2$ & $42.5 \pm 4.3$ & $29.9 \pm 4.9$ & $26.8 \pm 5.1$ & & & \\
\hline & 126 & $49.8 \pm 10.0$ & $38.2 \pm 3.9$ & $35.8 \pm 7.5$ & $23.6 \pm 3.5$ & & & \\
\hline \multirow[t]{5}{*}{ petunidins } & 50 & $0.2 \pm 0.1$ & $0.1 \pm 0.1$ & $0.2 \pm 0.1$ & $0.1 \pm 0.0$ & 0.0006 & 0.0386 & 0.9364 \\
\hline & 72 & $86.1 \pm 2.6$ & $44.2 \pm 8.2$ & $66.4 \pm 19.5$ & $43.2 \pm 9.4$ & & & \\
\hline & 92 & $271.0 \pm 39.7$ & $214.8 \pm 9.5$ & $219.7 \pm 23.3$ & $198.3 \pm 10.8$ & & & \\
\hline & 113 & $244.6 \pm 21.5$ & $264.4 \pm 27.7$ & $207.5 \pm 26.4$ & $201.2 \pm 18.0$ & & & \\
\hline & 126 & $272.0 \pm 14.3$ & $267.5 \pm 4.3$ & $234.6 \pm 40.8$ & $196.1 \pm 23.4$ & & & \\
\hline \multirow[t]{5}{*}{ peonidins } & 50 & $0.0 \pm 0.0$ & $0.0 \pm 0.0$ & $0.0 \pm 0.0$ & $0.0 \pm 0.0$ & 0.0002 & 0.0081 & 0.3947 \\
\hline & 72 & $63.6 \pm 5.6$ & $31.0 \pm 5.7$ & $46.4 \pm 13.5$ & $29.3 \pm 7.1$ & & & \\
\hline & 92 & $197.1 \pm 30.5$ & $115.5 \pm 22.2$ & $134.6 \pm 18.8$ & $116.6 \pm 9.6$ & & & \\
\hline & 113 & $181.4 \pm 19.8$ & $180.7 \pm 24.8$ & $132.8 \pm 20.9$ & $125.4 \pm 14.5$ & & & \\
\hline & 126 & $200.7 \pm 15.9$ & $184.3 \pm 6.6$ & $152.0 \pm 30.4$ & $125.6 \pm 15.2$ & & & \\
\hline \multirow[t]{5}{*}{ malvidins } & 50 & $0.8 \pm 0.2$ & $0.4 \pm 0.2$ & $0.8 \pm 0.1$ & $0.5 \pm 0.1$ & 0.0258 & 0.2348 & 0.7437 \\
\hline & 72 & $206.4 \pm 7.4$ & $113.9 \pm 18.0$ & $165.2 \pm 42.8$ & $112.3 \pm 22.7$ & & & \\
\hline & 92 & $587.5 \pm 6.2$ & $493.2 \pm 6.3$ & $503.5 \pm 31.9$ & $497.1 \pm 19.4$ & & & \\
\hline & 113 & $590.6 \pm 46.1$ & $663.5 \pm 42.7$ & $579.5 \pm 64.8$ & $566.7 \pm 7.4$ & & & \\
\hline & 126 & $670.5 \pm 48.1$ & $665.1 \pm 38.5$ & $601.7 \pm 72.1$ & $605.9 \pm 48.5$ & & & \\
\hline \multirow[t]{5}{*}{ trihydroxylated relative abundance } & 50 & $100.0 \pm 0.0$ & $100.0 \pm 0.0$ & $100.0 \pm 0.0$ & $100.0 \pm 0.0$ & 0.0001 & 0.0013 & 0.2360 \\
\hline & 72 & $82.3 \pm 1.6$ & $83.6 \pm 0.5$ & $83.4 \pm 0.3$ & $84.3 \pm 0.6$ & & & \\
\hline & 92 & $82.5 \pm 1.5$ & $86.3 \pm 1.8$ & $85.6 \pm 0.7$ & $86.5 \pm 0.7$ & & & \\
\hline & 113 & $82.9 \pm 1.4$ & $84.6 \pm 0.6$ & $86.1 \pm 0.5$ & $86.6 \pm 0.9$ & & & \\
\hline & 126 & $83.1 \pm 1.4$ & $84.6 \pm 0.4$ & $85.4 \pm 0.6$ & $87.0 \pm 0.2$ & & & \\
\hline
\end{tabular}


Table 2. Continued

\begin{tabular}{|c|c|c|c|c|c|c|c|c|}
\hline \multirow[b]{2}{*}{ compound } & \multirow[b]{2}{*}{ DAF } & \multicolumn{2}{|c|}{$+\mathrm{UV}-\mathrm{B}$} & \multicolumn{2}{|c|}{$-\mathrm{UV}-\mathrm{B}$} & \multirow[b]{2}{*}{$P_{(\mathrm{UV}-\mathrm{B})}$} & \multirow[b]{2}{*}{$P_{(\mathrm{ABA})}$} & \multirow[b]{2}{*}{$\left.P_{(\mathrm{UV}-\mathrm{B}} \times \mathrm{ABA}\right)$} \\
\hline & & $+\mathrm{ABA}$ & $-\mathrm{ABA}$ & $+\mathrm{ABA}$ & $-\mathrm{ABA}$ & & & \\
\hline \multirow[t]{5}{*}{ methoxylated relative abundance } & 50 & $82.3 \pm 1.7$ & $85.9 \pm 6.8$ & $79.4 \pm 1.7$ & $85.1 \pm 2.2$ & \multirow[t]{5}{*}{0.1949} & \multirow[t]{5}{*}{0.0302} & \multirow[t]{5}{*}{0.5740} \\
\hline & 72 & $76.5 \pm 1.3$ & $78.3 \pm 0.8$ & $77.9 \pm 0.5$ & $78.3 \pm 0.7$ & & & \\
\hline & 92 & $75.1 \pm 2.3$ & $76.2 \pm 1.1$ & $76.4 \pm 0.6$ & $78.1 \pm 1.3$ & & & \\
\hline & 113 & $76.8 \pm 1.4$ & $77.3 \pm 1.2$ & $79.4 \pm 0.3$ & $79.7 \pm 1.7$ & & & \\
\hline & 126 & $77.3 \pm 1.7$ & $77.2 \pm 0.5$ & $78.1 \pm 1.4$ & $81.4 \pm 0.8$ & & & \\
\hline
\end{tabular}

${ }^{a}+\mathrm{UV}-\mathrm{B}$ and $-\mathrm{UV}-\mathrm{B}$ treatments, combined with $+\mathrm{ABA}$ or $-\mathrm{ABA} . P_{(\mathrm{UV}-\mathrm{B})}, \mathrm{UV}-\mathrm{B}$ effect; $P_{(\mathrm{ABA})}, \mathrm{ABA}$ effect; $P_{(\mathrm{UV}-\mathrm{B} \times \mathrm{ABA})}, \mathrm{UV}-\mathrm{B} \times \mathrm{ABA}$ interaction effect. Values are the mean \pm SE.
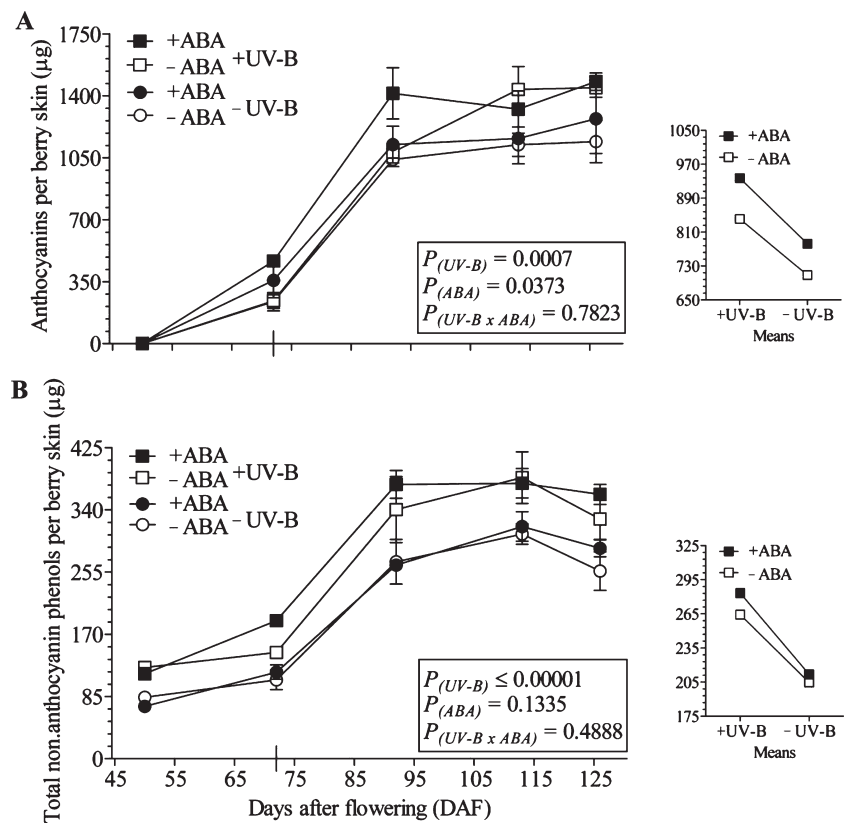

Figure 6. Anthocyanins (A) and total nonanthocyanin phenols (B) in micrograms per berry skin at 50, 72, 92, 113, and 126 DAF. + UV-B (squares) and -UV-B (circles) treatments, combined with $+\mathrm{ABA}$ (black) or $-\mathrm{ABA}$ (white) applications. $P_{(\mathrm{UV}-\mathrm{B})}$, UV-B effect; $P_{(\mathrm{ABA})}, \mathrm{ABA}$ effect; $\left.P_{(\mathrm{UV}-\mathrm{B}} \times \mathrm{ABA}\right), \mathrm{UV}-\mathrm{B} \times \mathrm{ABA}$ interaction effect. Values are the mean $\pm S E$, and veraison is indicated with a line at $72 \mathrm{DAF}$. The figures at the right are the overall means for the treatments.

at ca. 72 DAF (Figure 3). The +UV-B treatment reduced sugars per berry when combined with $+\mathrm{ABA}$ (interaction between UV-B and ABA, $P \leq 0.1$, Figure $3 \mathrm{~A}$ ). The $+\mathrm{UV}-\mathrm{B}$ treatment increased sugar concentration $\left({ }^{\circ} \mathrm{Brix}\right)$ independently of $\mathrm{ABA}$ treatment (Figure 3B). The effect on sugars (absolute amount per berry) began at $92 \mathrm{DAF}$ and was highest at harvest. The effect on ${ }^{\circ}$ Brix was apparent at veraison. Sugars were $11 \%$ greater per berry and $23.6 \%$ higher in ${ }^{\circ} \mathrm{Brix}$ in $+\mathrm{UV}-\mathrm{B} /+\mathrm{ABA}$ as compared with $-\mathrm{UV}-\mathrm{B} /-\mathrm{ABA}$ at veraison. However, at harvest $+\mathrm{UV}-\mathrm{B} /+$ $\mathrm{ABA}$ treatment gave a $23.3 \%$ lower value for sugars per berry, although for ${ }^{\circ}$ Brix the $+\mathrm{UV}-\mathrm{B} /+\mathrm{ABA}$ treatment result was very similar to that obtained for the $-\mathrm{UV}-\mathrm{B} /-\mathrm{ABA}$ treatment. These results are explained by Table 1 , which shows that berry growth (i.e., volume, FW, skin and pulp DW) at harvest was additively reduced by $+\mathrm{UV}-\mathrm{B}$ and $+\mathrm{ABA}$ (seed DW was diminished only by +UV-B, and the ratio of skin DW to berry DW was not affected). The $+\mathrm{UV}-\mathrm{B} /+\mathrm{ABA}$ treatment produced the smallest

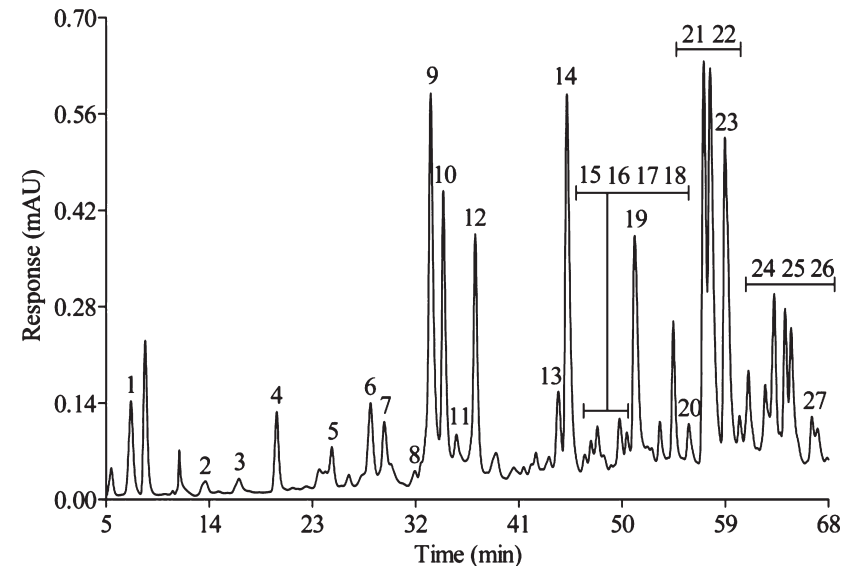

Figure 7. Typical HPLC-DAD chromatogram of berry skin nonanthocyanin phenols extracted with diethyl ether and ethyl acetate. Hydroxybenzoic acids and their derivatives: gallic acid (1); protocatechuic acid (2); vanillic acid (7); syringic acid (10); and methyl gallate (4). Hydroxycinnamic acids and their derivatives: caffeic acid (9); caftaric acid (3); and coutaric acid (12). Flavanols: procyanidin B3 (5); (+)-catechin (6); and procyanidins $(8,11$, and 15$)$. Flavonols: myricetin (27); myricetin 3-glucoside (17); myricetin 3-galactoside (18); myricetin 3- glucoside (19); kaempferol 3-galactoside (20); kaempferol 3-glucoside (26); quercetin 3-galactoside (22); quercetin 3-glucoside (23); and quercetin 3-rhamnoside (25). Dihydroflavonols (13, 14, 16, 21, and 24).

berries, with reductions of $32.5 \%$ in volume, $22.1 \%$ in $\mathrm{FW}$, and $31 \%$ in DW, as compared with - UV-B/-ABA.

ABA levels in the skin increased to $109.7 \%$ from 50 to $72 \mathrm{DAF}$ and then to $126.6 \%$ from 72 to $92 \mathrm{DAF}$, after which $\mathrm{ABA}$ decreased toward harvest, with a positive effect of $+\mathrm{ABA}$ (Figure 4). The $+\mathrm{UV}-\mathrm{B}$ irradiation treatment alone had no effects on overall $\mathrm{ABA}$ levels.

An anthocyanin characteristic HPLC-DAD profile at harvest is presented in Figure 5. Here, the general distribution by acylation was $35.7 \%$ acylated $(20.7 \%$ acetylated and $15 \%$ p-coumaroylated) and $64.3 \%$ nonacylated and that by anthocyanidins, $47.7 \%$ malvidin, $19 \%$ delphinidin, $18.2 \%$ petunidin, $12.4 \%$ peonidin, and $2.8 \%$ cyanidin (Table 2 ).

The total anthocyanins followed a sigmoid curve, with the major increases occurring from 72 to 92 DAF. +UV-B and $+\mathrm{ABA}$ additively augmented total anthocyanin accumulation until harvest. Thus, the $+\mathrm{UV}-\mathrm{B} /+\mathrm{ABA}$ treatment had 96.8 and $29.7 \%$ more total anthocyanins than $-U V-B /-A B A$ at 72 and $126 \mathrm{DAF}$, respectively (Figure 6A).

The anthocyanidins were differentially affected by UV-B and ABA. +UV-B increased all of the anthocyanidin glucosides, both 
Table 3. Nonanthocyanin Phenols (Micrograms per Berry Skin) and Flavonol Relative Abundance at 50, 72, 92, 113, and 126 $\mathrm{DAF}^{a}$

\begin{tabular}{|c|c|c|c|c|c|c|c|c|}
\hline \multirow[b]{2}{*}{ compound } & \multirow[b]{2}{*}{ DAF } & \multicolumn{2}{|c|}{$+U V-B$} & \multicolumn{2}{|c|}{-UV-B } & \multirow[b]{2}{*}{$P_{(\mathrm{UV}-\mathrm{B})}$} & \multirow[b]{2}{*}{$P_{(\mathrm{ABA})}$} & \multirow[b]{2}{*}{$\left.P_{(\mathrm{UV}-\mathrm{B}} \times \mathrm{ABA}\right)$} \\
\hline & & $+\mathrm{ABA}$ & $-\mathrm{ABA}$ & $+\mathrm{ABA}$ & $-\mathrm{ABA}$ & & & \\
\hline \multirow[t]{5}{*}{ flavonols } & 50 & $50.0 \pm 6.7$ & $54.6 \pm 3.3$ & $31.6 \pm 3.6$ & $26.0 \pm 1.1$ & \multirow[t]{5}{*}{0.00001} & \multirow[t]{5}{*}{0.0789} & \multirow[t]{5}{*}{0.3883} \\
\hline & 72 & $120.8 \pm 3.2$ & $84.9 \pm 3.3$ & $68.6 \pm 7.9$ & $62.9 \pm 10.5$ & & & \\
\hline & 92 & $233.4 \pm 17.5$ & $203.2 \pm 37.2$ & $146.9 \pm 8.3$ & $159.5 \pm 25.9$ & & & \\
\hline & 113 & $202.2 \pm 11.5$ & $214.0 \pm 25.0$ & $162.6 \pm 13.3$ & $148.8 \pm 16.1$ & & & \\
\hline & 126 & $207.7 \pm 9.8$ & $165.3 \pm 19.0$ & $139.6 \pm 12.3$ & $120.1 \pm 18.4$ & & & \\
\hline \multirow[t]{5}{*}{ dihydroflavonols } & 50 & $13.0 \pm 1.1$ & $16.2 \pm 1.5$ & $11.5 \pm 1.0$ & $11.5 \pm 0.8$ & \multirow[t]{5}{*}{0.00001} & \multirow[t]{5}{*}{0.5450} & \multirow[t]{5}{*}{0.6208} \\
\hline & 72 & $42.4 \pm 1.7$ & $34.5 \pm 1.2$ & $25.9 \pm 1.0$ & $24.1 \pm 3.4$ & & & \\
\hline & 92 & $89.9 \pm 2.0$ & $81.6 \pm 4.6$ & $68.2 \pm 6.2$ & $64.5 \pm 2.9$ & & & \\
\hline & 113 & $109.7 \pm 3.4$ & $109.5 \pm 6.3$ & $95.7 \pm 8.0$ & $95.9 \pm 2.3$ & & & \\
\hline & 126 & $95.7 \pm 1.7$ & $107.8 \pm 5.2$ & $95.0 \pm 6.4$ & $89.4 \pm 6.9$ & & & \\
\hline \multirow[t]{5}{*}{ flavanols } & 50 & $42.8 \pm 7.6$ & $40.0 \pm 12.5$ & $21.4 \pm 4.3$ & $36.4 \pm 3.7$ & \multirow[t]{5}{*}{0.0160} & \multirow[t]{5}{*}{0.8728} & \multirow[t]{5}{*}{0.6798} \\
\hline & 72 & $17.1 \pm 1.8$ & $19.3 \pm 1.5$ & $17.7 \pm 2.7$ & $15.7 \pm 0.9$ & & & \\
\hline & 92 & $32.7 \pm 4.1$ & $37.4 \pm 4.9$ & $34.2 \pm 1.7$ & $30.2 \pm 2.6$ & & & \\
\hline & 113 & $40.5 \pm 6.2$ & $40.7 \pm 4.6$ & $35.9 \pm 2.2$ & $41.4 \pm 2.5$ & & & \\
\hline & 126 & $39.8 \pm 2.1$ & $32.8 \pm 5.9$ & $32.5 \pm 4.3$ & $24.2 \pm 1.3$ & & & \\
\hline \multirow[t]{5}{*}{ hydroxycinnamic acids } & 50 & $9.9 \pm 0.3$ & $13.5 \pm 0.3$ & $6.6 \pm 0.1$ & $9.7 \pm 0.9$ & \multirow[t]{5}{*}{0.0978} & \multirow[t]{5}{*}{0.4396} & \multirow[t]{5}{*}{0.8618} \\
\hline & 72 & $3.8 \pm 0.1$ & $3.0 \pm 0.6$ & $2.7 \pm 0.2$ & $2.2 \pm 0.3$ & & & \\
\hline & 92 & $9.7 \pm 0.9$ & $9.0 \pm 0.1$ & $8.0 \pm 1.4$ & $7.6 \pm 1.0$ & & & \\
\hline & 113 & $12.3 \pm 0.3$ & $10.8 \pm 0.7$ & $12.8 \pm 1.2$ & $10.8 \pm 1.5$ & & & \\
\hline & 126 & $9.2 \pm 0.6$ & $11.2 \pm 1.7$ & $11.0 \pm 1.6$ & $12.3 \pm 1.5$ & & & \\
\hline \multirow[t]{5}{*}{ hydroxybenzoic acids } & 50 & $0.3 \pm 0.0$ & $0.4 \pm 0.0$ & $0.4 \pm 0.0$ & $0.2 \pm 0.1$ & \multirow[t]{5}{*}{0.0571} & \multirow[t]{5}{*}{0.4466} & \multirow[t]{5}{*}{0.6907} \\
\hline & 72 & $4.4 \pm 0.3$ & $3.4 \pm 0.4$ & $3.4 \pm 0.2$ & $2.7 \pm 0.2$ & & & \\
\hline & 92 & $9.1 \pm 0.7$ & $9.2 \pm 0.5$ & $7.3 \pm 0.9$ & $7.3 \pm 0.6$ & & & \\
\hline & 113 & $11.4 \pm 0.2$ & $9.1 \pm 0.5$ & $10.2 \pm 0.9$ & $9.9 \pm 0.3$ & & & \\
\hline & 126 & $8.7 \pm 0.6$ & $10.3 \pm 0.3$ & $9.6 \pm 0.8$ & $10.2 \pm 0.9$ & & & \\
\hline \multirow[t]{5}{*}{ flavonol relative abundance } & 50 & $43.1 \pm 5.2$ & $44.4 \pm 5.7$ & $44.5 \pm 4.0$ & $31.2 \pm 1.5$ & \multirow[t]{5}{*}{0.0005} & 0.0370 & 0.8673 \\
\hline & 72 & $64.1 \pm 0.7$ & $58.5 \pm 1.1$ & $57.7 \pm 2.0$ & $57.8 \pm 2.2$ & & & \\
\hline & 92 & $62.1 \pm 1.6$ & $59.0 \pm 3.1$ & $55.5 \pm 2.7$ & $58.5 \pm 3.6$ & & & \\
\hline & 113 & $53.7 \pm 0.2$ & $55.5 \pm 1.5$ & $51.2 \pm 1.7$ & $48.2 \pm 3.1$ & & & \\
\hline & 126 & $57.5 \pm 0.7$ & $50.2 \pm 1.6$ & $48.4 \pm 2.9$ & $46.4 \pm 2.7$ & & & \\
\hline
\end{tabular}

${ }^{a}+\mathrm{UV}-\mathrm{B}$ and $-\mathrm{UV}-\mathrm{B}$ treatments, combined with $+\mathrm{ABA}$ or $-\mathrm{ABA} . P_{(\mathrm{UV}-\mathrm{B})}, \mathrm{UV}-\mathrm{B}$ effect; $P_{(\mathrm{ABA})}, \mathrm{ABA}$ effect; $P_{(\mathrm{UV}-\mathrm{B} \times \mathrm{ABA})}, \mathrm{UV}-\mathrm{B} \times \mathrm{ABA}$ interaction effect. Values are the mean \pm SE.

acylated and nonacylated, whereas + ABA only augmented total nonacylated, total delphinidin, cyanidin, petunidin, and peonidin. In +UV-B/+ABA treatment, relative to the $-\mathrm{UV}-\mathrm{B} /-\mathrm{ABA}$ treatment, both at harvest, cyanidin increased the most (110\%), whereas delphinidin, petunidin, and peonidin increased ca. 50\%, with malvidin increasing only by ca. $10 \%$. Consequently, +UV-B and $+\mathrm{ABA}$ additively reduced the relative abundance of total trihydroxylated anthocyanins (petunidin-, delphinidin-, and malvidin-derived), whereas $+\mathrm{ABA}$ decreased the proportion of total methoxylated anthocyanins (peonidin-, petunidin-, and malvidin-derived; Table 2).

A nonanthocyanin phenol representative HPLC-DAD profile at harvest is shown in Figure 7. The profile changes markedly from 50 to 126 DAF: for flavonols, dihydroflavonols (direct precursors of flavonols) and hydroxybenzoic acids, for example, 40.5, 13.5, and $0.4 \%$, respectively, of the total at $50 \mathrm{DAF}$, and by $126 \mathrm{DAF}$ this had increased to $50.9,31.8$, and $3.2 \%$, respectively. The flavanols and hydroxycinnamic acids diminished from 35.6 and $10 \%$, respectively, of the total to 10.4 and $3.6 \%$, respectively (Table 3 ).

The total nonanthocyanin phenols followed a sigmoid curve with the higher increases occurring from 50 to $92 \mathrm{DAF}$. The +UV-B treatment increased nonanthocyanin phenol accumulation through to harvest, whereas $+\mathrm{ABA}$ alone treatment had no effects on overall amount. In contrast, $+\mathrm{UV}-\mathrm{B} /+\mathrm{ABA}$ treatment gave 75 and $40.9 \%$ higher levels than $-U V-B /-A B A$ at 72 and $126 \mathrm{DAF}$, respectively (Figure 6B). The +UV-B alone treatment increased all of the subgroup totals, whereas $+\mathrm{ABA}$ alone increased only the total flavonols $(P \leq 0.1$, Table 3$)$. 
A

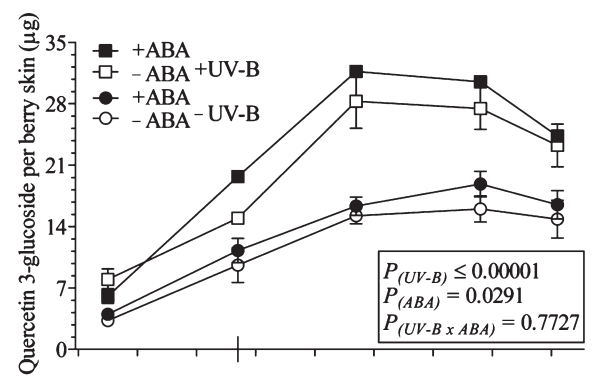

B

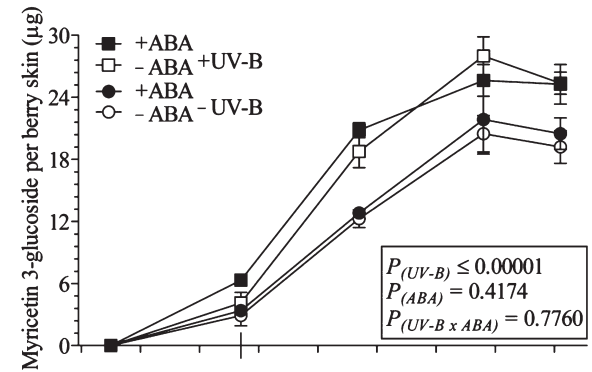

C

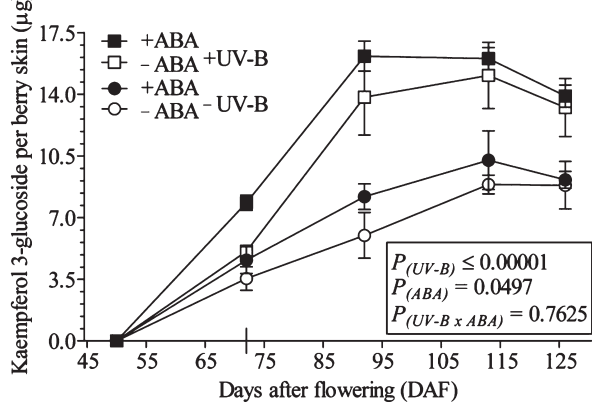

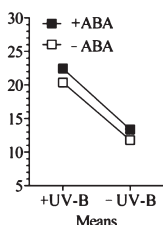
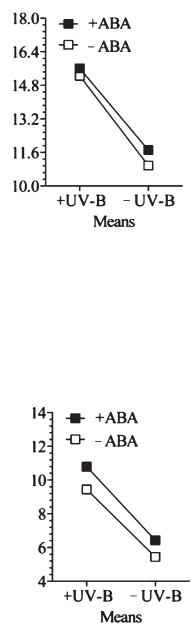

Figure 8. Flavonol glucosides ( $\mu \mathrm{g}$ per berry skin) quercetin (A), myricetin (B), and kaempferol (C) at 50, 72, 92, 113, and 126 DAF. +UV-B (squares) and -UV-B (circles) treatments, combined with $+\mathrm{ABA}$ (black) or $-\mathrm{ABA}$ (white) applications. $P_{(\mathrm{UV}-\mathrm{B})}$, UV-B effect; $P_{(\mathrm{ABA})}, \mathrm{ABA}$ effect; $\left.P_{(\mathrm{UV}-\mathrm{B}} \times \mathrm{ABA}\right), \mathrm{UV}-\mathrm{B} \times \mathrm{ABA}$ interaction effect. Values are the mean $\pm S E$, and veraison is indicated with a line at $72 \mathrm{DAF}$. The figures at the right are the overall means for the treatments.

Specifically, +UV-B treatment increased all of the flavonols detected. Figure 8 shows the main flavonols, quercetin, myricetin, and kaempferol glucosides, whereas flavanols, $(+)$-catechin and procyanidin B3, are shown in Figure 9, and hydroxybenzoic acids, gallic, protocatechuic, and vanillic acids, are shown in Figure 10. Data are not shown for dihydroflavonols.

The +UV-B alone treatment did not affect the most abundant hydroxybenzoic acid (syringic acid; data not shown), nor did it affect the main hydroxycinnamic acids (caffeic and coutaric acids; Figure 11). In contrast, the $+\mathrm{ABA}$ treatment increased specifically the quercetin and kaempferol glucosides (Figure 8A,C), gallic and protocatechuic acids (Figure 10A,B) and one of the dihydroflavonols that was detected (peak 16; Figure 7).

The flavonol relative abundance was increased by the +UV$\mathrm{B} /+\mathrm{ABA}$ treatment, that is, $23.9 \%$ higher than $-\mathrm{UV}-\mathrm{B} /-\mathrm{ABA}$ at harvest (Table 3).

\section{DISCUSSION}

The treatment that combined $+\mathrm{UV}-\mathrm{B} /+\mathrm{ABA}$ gave increased sugar loading to the berry until veraison, although by the time of harvest that treatment had decreased sugars on a per berry basis;

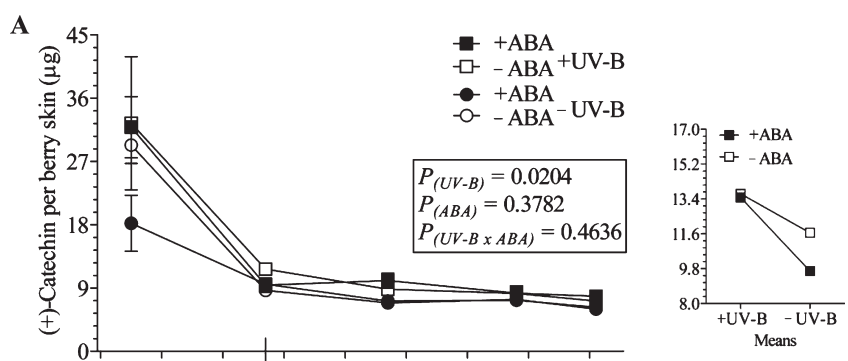

B

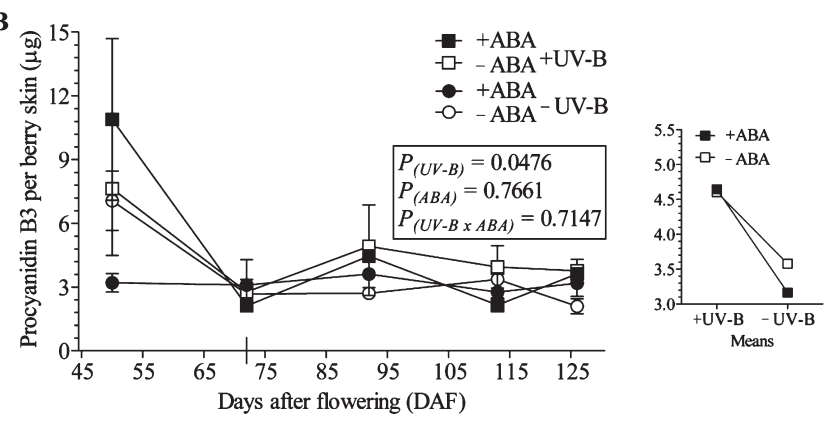

Figure 9. Flavanol ( $\mu$ g per berry skin) $(+)$-catechin (A) and procyanidin B3 (B) at 50, 72, 92, 113, and 126 DAF. +UV-B (squares) and - UV-B (circles) treatments, combined with $+\mathrm{ABA}$ (black) or $-\mathrm{ABA}$ (white) applications. $P_{(\mathrm{UV}-\mathrm{B})}$, UV-B effect; $P_{(\mathrm{ABA})}, \mathrm{ABA}$ effect;

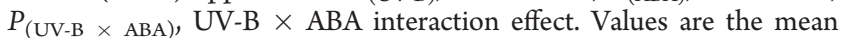
$\pm \mathrm{SE}$, and veraison is indicated with a line at $72 \mathrm{DAF}$. The figures at the right are the overall means for the treatments.

for example, there was an appreciable reduction in hexose loading to the grape berries between veraison and harvest, although the ${ }^{\circ} \mathrm{Brix}$ was not affected because of a diminution in individual berry size. Therefore, the $+\mathrm{UV}-\mathrm{B} /+\mathrm{ABA}$ treatment hastened the onset of ripening, but diminished subsequent fruit growth after $92 \mathrm{DAF}$ and presumably also reduced the flow of carbohydrates into the berry. In a previous work with pot-grown Malbec grapevines, it was also found that $+\mathrm{ABA}$ treatments increased monosaccharide (glucose and fructose) accumulation in berries and roots up to veraison. ${ }^{14}$ Others have also shown that $+\mathrm{ABA}$ can enhance sugar accumulation in berries, possibly because $A B A$ stimulates the activity of various acid invertases and hexose transporters. ${ }^{24,25}$

The berry growth reduction after $92 \mathrm{DAF}$ in $+\mathrm{UV}-\mathrm{B} /+\mathrm{ABA}$ could be related to a decrease in berry elasticity, because another experiment showed that $\mathrm{ABA}$ application to in vitro cultured grape berries diminished berry elasticity and growth. ${ }^{26}$ Thus, berries on $+\mathrm{UV}-\mathrm{B} /+\mathrm{ABA}$-treated plants may approach this limit earlier due to an ABA-induced advancement of ripening. Also, $A B A$ appears to induce a faster degradation of a vacuolar invertase protein that plays a key role in berry hexose accumulation, ${ }^{27}$ and this could explain our finding of less sugar accumulation per berry after $92 \mathrm{DAF}$.

Even though the uptake of $\mathrm{ABA}$ into the berry through the waxy cuticle is likely to be an inefficient process, as this structure forms an effective barrier and $A B A$ may be rapidly metabolized by vegetative tissue enzymes, $A B A$ levels were increased in the berry skin as a result of exogenous $+\mathrm{ABA}$. Although the $+\mathrm{UV}-\mathrm{B}$ alone treatment did not affect $A B A$ levels in the berry skin tissues, the $+\mathrm{UV}-\mathrm{B} /+\mathrm{ABA}$ treatment produced the highest berry skin $\mathrm{ABA}$ levels. In a previous work with grape leaf tissues $A B A$ levels were increased 2.7-fold by + UV-B and 12.5-fold in leaf tissue subjected to $+\mathrm{UV}-\mathrm{B} /+\mathrm{ABA}{ }^{8}{ }^{8}$ Thus, the berry is relatively nonresponsive 

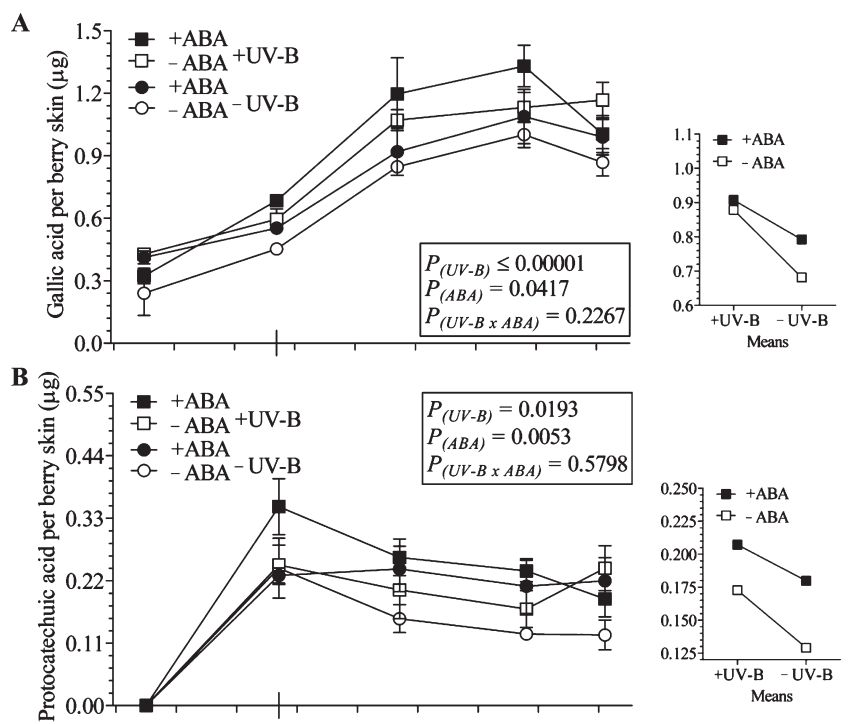

C
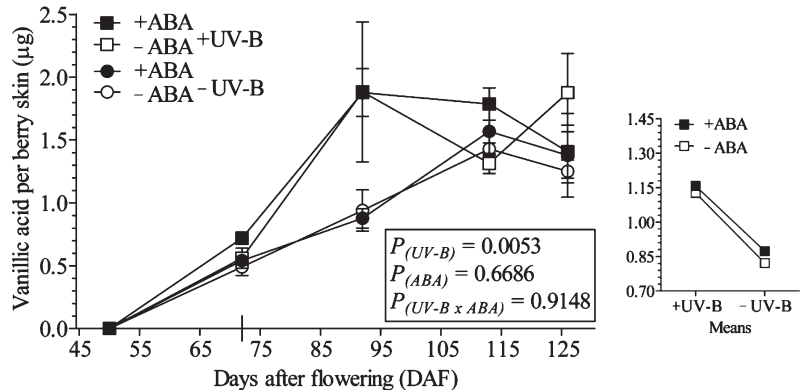

Figure 10. Hydroxybenzoic acids ( $\mu$ g per berry skin) gallic acid (A), protocatechuic acid (B), and vanillic acid (C) at 50, 72, 92, 113, and $126 \mathrm{DAF}$. +UV-B (squares) and -UV-B (circles) treatments, combined with $+\mathrm{ABA}$ (black) or $-\mathrm{ABA}$ (white) applications. $P_{(\mathrm{UV}-\mathrm{B})}$, UV$\mathrm{B}$ effect; $P_{(\mathrm{ABA})}, \mathrm{ABA}$ effect; $\left.P_{(\mathrm{UV}-\mathrm{B}} \times \mathrm{ABA}\right), \mathrm{UV}-\mathrm{B} \times \mathrm{ABA}$ interaction effect. Values are the mean $\pm S E$, and veraison is indicated with a line at $72 \mathrm{DAF}$. The figures at the right are the overall means for the treatments.

to UV-B treatment in terms of enhancing berry skin ABA levels, possibly as a consequence of the UV-B filtering by phenols that are much more abundant in berry skins.

$\mathrm{ABA}$ increased in all treatments gradually before veraison and then more rapidly as the ripening proceeded, up to $92 \mathrm{DAF}$. ABA levels then decreased to very low levels toward harvest. In other experiments with field-grown Cabernet Sauvignon, the berry's ABA also increased at veraison, reaching the maximum ca. 2 weeks after the onset of ripening and then declining. ${ }^{13}$ Most studies have shown that grape berry $\mathrm{ABA}$ increases at about the time of veraison, although the reported timing of this increase in relation to veraison varies somewhat. ${ }^{28}$ Fruits such as grape are considered to be nonclimacteric, and the physiological controls for the onset of ripening are still poorly understood. ${ }^{26}$ This contrasts with climacteric fruits, in which the control of ripening is predominately mediated by ethylene. Finally, it is not known whether the peak in grape berry $\mathrm{ABA}$ is a consequence of the initiation of ripening due to the osmotic stress caused by the rapid increase in berry sugar accumulation or if that ABA peak is part of the mechanism essential for triggering ripening. It should be noted here that transcript abundance of 9-cis-epoxycarotenoid dioxygenase (NCED1) was increased significantly 1 week after veraison in Cabernet Sauvignon, and the authors proposed that
A
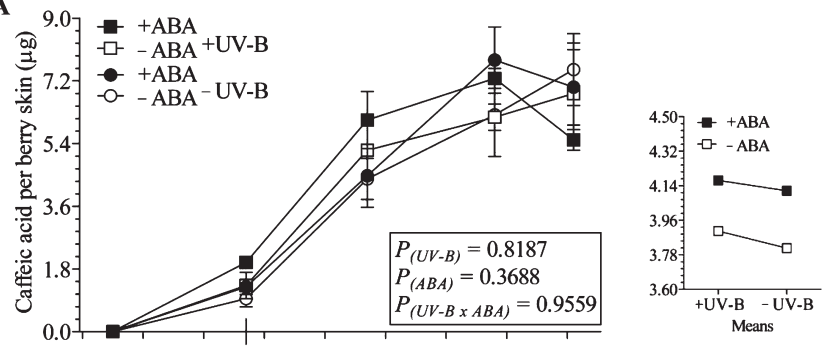

B

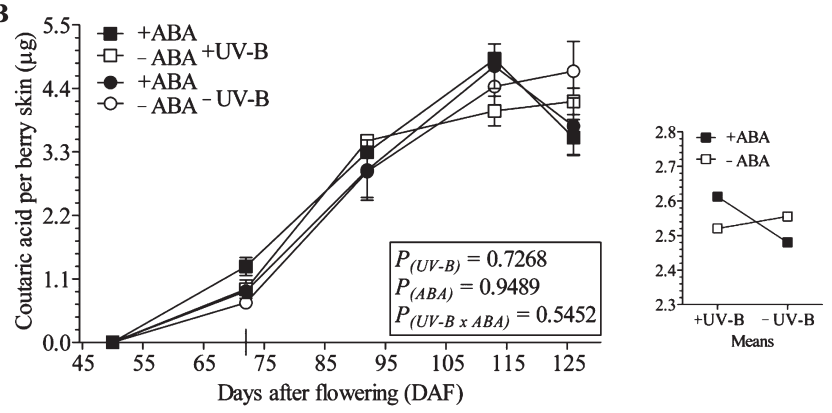

Figure 11. Hydroxycinnamic acids and their derivatives ( $\mu$ g per berry skin), caffeic acid (A) and coutaric acid (B), at 50, 72, 92, 113, and 126 DAF. +UV-B (squares) and -UV-B (circles) treatments, combined with $+\mathrm{ABA}$ (black) or $-\mathrm{ABA}$ (white) applications. $P_{(\mathrm{UV}-\mathrm{B})}$, UV-B effect; $P_{(\mathrm{ABA})}, \mathrm{ABA}$ effect; $\left.P_{(\mathrm{UV}-\mathrm{B}} \times \mathrm{ABA}\right), \mathrm{UV}-\mathrm{B} \times \mathrm{ABA}$ interaction effect. Values are the mean $\pm \mathrm{SE}$, and veraison is indicated with a line at $72 \mathrm{DAF}$. The figures at the right are the overall means for the treatments.

grape berry $\mathrm{ABA}$ biosynthesis was regulated primarily by this gene in berries and not by $\mathrm{ABA}$ transported into the berry from an external source such as leaves or roots. ${ }^{29}$

Anthocyanins are postulated to play an important role in plant reproduction by attracting pollinators and seed dispersers, providing a high contrast between background foliage and fruits, ${ }^{30}$ as well as providing protection to solar UV-B in conjunction with other flavonoids. ${ }^{9}$ The present study demonstrates a positive effect of $+\mathrm{UV}-\mathrm{B}$ and $+\mathrm{ABA}$ on anthocyanin accumulation prior to veraison and also on maintaining high anthocyanin levels until harvest. This effect of $+\mathrm{ABA}$ has been also reported and can be explained by activation of phenylpropanoid and flavonoid biosynthetic pathway enzymes (Figure 1). ${ }^{31}$ Also, genes involved in the acylation and transport of anthocyanins into the skin cell vacuoles show a peak of activity during veraison. ${ }^{24}$ UV-B was found to up-regulate phenolic key enzymes such as PAL and CHS in grape leaves, ${ }^{11}$ and others have reported a significantly higher total anthocyanin concentration in sun-exposed Shiraz berries, relative to shaded fruit shortly after veraison, although the differences were not maintained at harvest. ${ }^{32}$

During the ripening period the nonacylated anthocyanins were more abundant than the acylated forms, malvidin being the main anthocyanidin as in most grapevine cultivars. ${ }^{33}$ The predominance of trihydroxylated anthocyanins in grape berries has been associated with higher ratios of flavonoid $3^{\prime}, 5^{\prime}$-hydroxylase $\left(\mathrm{F} 3^{\prime} 5^{\prime} \mathrm{H}\right)$ to flavonoid $3^{\prime}$-hydroxylase $\left(\mathrm{F} 3^{\prime} \mathrm{H}\right)$ transcription activity and higher levels of $o$-methyltransferase (OMT) transcripts. ${ }^{33-35}$ Anthocyanins appeared ca. 120 million years ago, ${ }^{36}$ and most species, including grapevines, have later acquired the ability of synthesizing (anthocyanidin) blue pigments, with a higher number of substituted and methoxylated groups in the flavan nucleus. That is, $\mathrm{F}^{\prime} \mathrm{H}$ (responsible for red anthocyanin 
synthesis) is ancestral to $\mathrm{F}^{\prime} 5^{\prime} \mathrm{H}$ (that leads to the conversion of red into blue anthocyanins). Assuming that the level of UV-B reaching the vegetation at that time was considerably higher than today, it would be probable that protective mechanisms and their corresponding signaling pathways evolved (and thus should be present) in all land plants. Although the anthocyanin profile is primarily a genetic characteristic unique to each grapevine cultivar, $^{37}$ the present study shows that $+\mathrm{UV}-\mathrm{B}$ and $+\mathrm{ABA}$ additively reduced the proportion of trihydroxylated anthocyanins, suggesting that $\mathrm{F}^{\prime} \mathrm{H}$ was relatively more active than $\mathrm{F}^{\prime} 5^{\prime} \mathrm{H}$, whereas $+\mathrm{ABA}$ alone reduced the proportion of methoxylated forms, indicating that OMT was less active. This acclimation shift in anthocyanin composition has also been shown in sun-exposed fruits, where the proportion of trihydroxylated anthocyanins was reduced as compared with shaded ones. ${ }^{38}$ It has also been demonstrated that the plant water status (presumably affecting ABA levels) may alter the degree of anthocyanin hydroxylation and methoxylation in grape berries. ${ }^{39}$ The most oxidized (trihydroxylated and methoxylated) anthocyanins have less antioxidant power, whereas the oxygen radical absorbing capacity (ORAC) of cyanidin 3-glucoside is the highest. ${ }^{48}$ In the present experiment the total anthocyanins were additively increased by $+\mathrm{UV}-\mathrm{B}$ and $+\mathrm{ABA}$, but cyanidin was increased the most. Increases in delphinidin, cyanidin, petunidin, and peonidin levels have also been found in response to partial rootzone drying (PRD), a viticulture practice that allegedly maintains relatively high levels of ABA in the xylem stream, ${ }^{20}$ whereas malvidin was unaffected and methoxylated anthocyanins were reduced by water restriction. ${ }^{39}$

Solar UV-B also induces an accumulation of total nonanthocyanin phenols and maintains those high levels until harvest. In contrast, +ABA treatment increased specific compounds, mainly flavonols and hydroxybenzoic acids. Furthermore, the flavonol relative abundance was additively increased by $+\mathrm{UV}-\mathrm{B}$ and $+\mathrm{ABA}$ treatments, and this may occur because flavonol synthase (FLS) was more active. In a previous work with grape leaves, quercetin and kaempferol were also increased by $+\mathrm{UV}-\mathrm{B}$ and + ABA more markedly than the other detected phenols. ${ }^{8}$ Others have also found enhanced synthesis of quercetin and kaempferol in grape leaves ${ }^{41}$ and total flavonoids in grape berries ${ }^{10}$ exposed to UV-B.

Flavonols were most abundant at the different ripening stages, and flavanols represented only $10.4 \%$ of the total at harvest. Flavanols accumulate mainly before veraison and then decreased toward harvest mainly because of polymerization that diminished extractability. ${ }^{19}$ +UV-B treatment produced higher flavanol levels, and $+\mathrm{ABA}$ treatment did not affect the decline of these compounds during ripening, as has been previously found. ${ }^{24}$

In conclusion, solar UV-B at high altitudes and exogenously applied ABA hastened the onset of ripening (sugar and phenol accumulation) and further decreased berry growth and sugars in an absolute amount (per berry), without affecting the sugar concentration at harvest. ABA levels in the berry skin were markedly increased from the onset of ripening to 20 days thereafter and then decreased toward harvest. This lends support to the hypothesis that the $\mathrm{ABA}$ does control berry ripening in grape. Most of the phenols in berry skin were increased by +UV-B treatment, with an additional increase when ABA was applied, and the levels of these phenols were maintained until harvest. Both +UV-B and + ABA treatments changed the anthocyanin and nonanthocyanin profiles, increasing the proportion of those phenols with high antioxidant capacity. The $+\mathrm{ABA}$ treatment did not produce the same effect as the +UV-B treatment, which indicates not only that the UV-B signal is mediated by ABA but also that other factors are likely involved.

\section{AUTHOR INFORMATION}

\section{Corresponding Author}

*Phone: 54-261-4135010. Fax: 54-261-4960469. E-mail: rbottini@ fca.uncu.edu.ar.

\section{Funding Sources}

This work was supported by Consejo Nacional de Investigaciones Científicas y Técnicas (CONICET, PAE-PID 2007-00149 to R. B.), Secretaría de Ciencia y Técnica de la Nación (SECyT, PICT 2008-1666 to R.B.), Secretaría de Ciencia y Técnica de la Universidad Nacional de Cuyo, and Bodega Catena-Zapata.

\section{ACKNOWLEDGMENT}

Special thanks to L. Bolcato, M. Fucili, and R. Alonso for their technical assistance.

\section{ABBREVIATIONS USED}

$\mathrm{ABA}$, abscisic acid; $+\mathrm{ABA}$, plus $\mathrm{ABA}$ treatment; $-\mathrm{ABA}$, minus $\mathrm{ABA}$ treatment; $\mathrm{ANR}$, anthocyanidin reductase; ${ }^{\circ} \mathrm{Brix}$, relative concentration of sugars; $\mathrm{CHI}$, chalcone isomerase; $\mathrm{CHS}$, chalcone synthase; DAF, days after flowering; DFR, dihydroflavonol reductase; DW, dry weight; $\mathrm{F} 3 \mathrm{H}$, flavanone 3-hydroxylase; $\mathrm{F} 3^{\prime} \mathrm{H}$, flavonoid $3^{\prime}$-hydroxylase; F3 $5^{\prime} \mathrm{H}$, flavonoid $3^{\prime}, 5^{\prime}$-hydroxylase; FLS, flavonol synthase; FW, fresh weight; GC-EIMS, capillary gas chromatography-electron impact mass spectrometry; HPLC-DAD, high-performance liquid chromatography equipment with photodiode array detector; LAR, leucoanthocyanidin reductase; LDOX, leucoanthocyanidin dioxygenase; OMT, $o$-methyltransferase; ORAC, oxygen radical absorbing capacity; PAL, phenylalanine ammonia-lyase; PAR, photosynthetically active radiation; $\mathrm{PE}$, polyester; $\mathrm{PET}$, polyethylene; $\mathrm{PRD}$, partial rootzone drying; UFGT, flavonoid 3-glucosyltransferase; UV-B, ultraviolet-B radiation; +UV-B, full UV-B treatment; -UV-B, minus UV-B treatment.

\section{REFERENCES}

(1) Paul, N. D.; Gwynn-Jones, D. Ecological roles of solar UV radiation: towards an integrated approach. Trends Ecol. Evol. 2003, 18, $48-55$.

(2) Berli, F.; D’Angelo, J.; Cavagnaro, B.; Bottini, R.; Wuilloud, R.; Silva, M. F. Phenolic composition in grape (Vitis vinifera L. cv. Malbec) ripened with different solar UV-B radiation levels by capillary zone electrophoresis. J. Agric. Food Chem. 2008, 56, 2892-2898.

(3) Caldwell, M. M.; Flint, S. D. Uses of biological spectral weighting functions and the need of scaling for the ozone reduction problem. Plant Ecol. 1997, 128, 66-76.

(4) Jansen, M. A. K.; Gaba, V.; Greenberg, B. M. Higher plants and UV-B radiation: balancing damage, repair and acclimation. Trends Plant Sci. 1998, 3, 131-135.

(5) Krupa, S. V.; Jäger, H. J. Adverse effects of elevated levels of ultraviolet (UV)-B radiation and ozone $\left(\mathrm{O}_{3}\right)$ on crop growth and productivity. In Global Climate Change and Agricultural Production: Direct and Indirect Effects of Changing Hydrological, Pedological and Plant Physiological Processes; Bazzaz, F., Sombroek, W., Eds.; Wiley: Chichester, U.K., 1996; pp 141-169.

(6) Jenkins, G. I. Signal transduction in responses to UV-B radiation. Annu. Rev. Plant Biol. 2009, 60, 407-431. 
(7) Olsson, L. C.; Veit, M.; Weissenböck, G.; Bornman, J. F. Differential flavonoid response to enhanced UV-B radiation in Brassica napus. Phytochemistry 1998, 49, 1021-1028.

(8) Berli, F. J.; Moreno, D.; Piccoli, P.; Hespanhol-Viana, L.; Silva, M. F.; Bressan-Smith, R.; Cavagnaro, J. B.; Bottini, R. Abscisic acid is involved in the response of grape (Vitis vinifera $\mathrm{L}$.) $\mathrm{cv}$. Malbec leaf tissues to ultraviolet-B radiation by enhancing ultraviolet-absorbing compounds, antioxidant enzymes and membrane sterols. Plant Cell Environ. 2010, 33, 1-10.

(9) Solovchenko, A.; Schmitz-Eiberger, M. Significance of skin flavonoids for UV-B-protection in apple fruits. J. Exp. Bot. 2003, 54, 1977-1984.

(10) Schultz, H. R. Climate change and viticulture: a European perspective on climatology, carbon dioxide and UV-B effects. Aust. J. Grape Wine Res. 2000, 6, 2-12.

(11) Pontin, M. A.; Piccoli, P. N.; Francisco, R.; Bottini, R.; MartinezZapater, J. M.; Lijavetzky, D. Transcriptome changes in grapevine (Vitis vinifera L.) cv. Malbec leaves induced by ultraviolet-B radiation. BMC Plant Biol. 2010, 10.

(12) Seki, M.; Ishida, J.; Narusaka, M.; Fujita, M.; Nanjo, T.; Umezawa, T.; Kamiya, A.; Nakajima, M.; Enju, A.; Sakurai, T.; Satou, M.; Akiyama, K.; Yamaguchi-Shinozaki, K.; Carninci, P.; Kawai, J.; Hayashizaki, Y.; Shinozaki, K. Monitoring the expression pattern of around 7,000 Arabidopsis genes under ABA treatments using a full-length cDNA microarray. Funct. Integr. Genomics 2002, 2, 282-291.

(13) Wheeler, S.; Loveys, B.; Ford, C.; Davies, C. The relationship between the expression of abscisic acid biosynthesis genes, accumulation of abscisic acid and the promotion of Vitis vinifera L. berry ripening by abscisic acid. Aust. J. Grape Wine Res. 2009, 15, 195-204.

(14) Moreno, D.; Berli, F.; Piccoli, P.; Bottini, R. Gibberellins and abscisic acid promote carbon allocation in roots and berries of grapevines. J. Plant Growth Regul. 2010, DOI: 10.1007/s00344-010-9186-4.

(15) Croteau, R.; Kutchan, T. M.; Lewis, N. G. Natural products (secondary metabolites). In Biochemistry and Molecular Biology of Plants; Buchanan, B., Gruissem, W., Jones, R., Eds.; American Society of Plant Biologists: Rockville, MD, 2000; pp 1250-1268.

(16) Cheynier, V. Polyphenols in foods are more complex than often thought. Am. J. Clin. Nutr. 2005, 81, 223-229.

(17) Boss, P. K.; Davies, C.; Robinson, S. P. Analysis of the expression of anthocyanin pathway genes in developing Vitis vinifera L. cv Shiraz grape berries and the implications for pathway regulation. Plant Physiol. 1996, 111, 1059-1066.

(18) Price, S. F.; Breen, P. J.; Valladao, M.; Watson, B. T. Cluster sun exposure and quercetin in Pinot noir grapes and wine. Am. J. Enol. Vitic. 1995, 46, 187-194.

(19) Kennedy, J. A.; Hayasaka, Y.; Vidal, S.; Waters, E. J.; Jones, G. P. Composition of grape skin proanthocyanidins at different stages of berry development. J. Agric. Food Chem. 2001, 49, 5348-5355.

(20) Stoll, M.; Loveys, B.; Dry, P. Hormonal changes induced by partial rootzone drying of irrigated grapevine. J. Exp. Bot. 2000, 51 1627-1634.

(21) Coombe, B. J. Growth stages of the grapevine: adoption of a system for identifying grapevine growth stages. Aust. J. Grape Wine Res. 1995, 1, 104-110.

(22) Quiroga, A. M.; Berli, F. J.; Moreno, D.; Cavagnaro, J. B.; Bottini, R. Abscisic acid sprays significantly increase yield per plant in vineyard-grown wine grape (Vitis vinifera L.) cv. Cabernet Sauvignon through increased berry set with no negative effects on anthocyanin content and total polyphenol index of both juice and wine. J. Plant Growth Regul. 2009, 28, 28-35.

(23) Fanzone, M.; Pena-Neira, A.; Jofre, V.; Assof, M.; Zamora, F. Phenolic characterization of malbec wines from mendoza province (Argentina). J. Agric. Food Chem. 2010, 58, 2388-2397.

(24) Koyama, K.; Sadamatsu, K.; Goto-Yamamoto, N. Abscisic acid stimulated ripening and gene expression in berry skins of the Cabernet Sauvignon grape. Funct. Integr. Genomics 2010, 10, 367-381.
(25) Hayes, M. A.; Feechan, A.; Dry, I. B. Involvement of abscisic acid in the coordinated regulation of a stress-inducible hexose transporter (vvht5) and a cell wall invertase in grapevine in response to biotrophic fungal infection. Plant Physiol. 2010, 153, 211-221.

(26) Gambetta, G. A.; Matthews, M. A.; Shaghasi, T. H.; McElrone, A. J.; Castellarin, S. D. Sugar and abscisic acid signaling orthologs are activated at the onset of ripening in grape. Planta 2010, 232, 219-234.

(27) Giribaldi, M.; Gény, L.; Delrot, S.; Schubert, A. Proteomic analysis of the effects of ABA treatments on ripening Vitis vinifera berries. J. Exp. Bot. 2010, 61, 2447-2458.

(28) Lacampagne, S.; Gagné, S.; Gény, L. Involvement of abscisic acid in controlling the proanthocyanidin biosynthesis pathway in grape skin: new elements regarding the regulation of tannin composition and leucoanthocyanidin reductase (LAR) and anthocyanidin reductase (ANR) activities and expression. J. Plant Growth Regul. 2010, 29, 81-90.

(29) Deluc, L. G.; Quilici, D. R.; Decendit, A.; Grimplet, J.; Wheatley, M. D.; Schlauch, K. A.; Mèrillon, J. M.; Cushman, J. C.; Cramer, G. R. Water deficit alters differentially metabolic pathways affecting important flavor and quality traits in grape berries of Cabernet Sauvignon and Chardonnay. BMC Genomics 2009, 10, 212-245.

(30) Grotewold, E. The genetics and biochemistry of floral pigments. Annu. Rev. Plant Biol. 2006, 57, 761-80.

(31) Owen, S. J.; Lafond, M. D.; Bowen, P.; Bogdanoff, C.; Usher, K.; Abrams, S. R. Profiles of abscisic acid and its catabolites in developing Merlot grape (Vitis vinifera) berries. Am. J. Enol. Viticult. 2009, 60, 277-284.

(32) Haselgrove, L.; Botting, D.; Van Heeswijck, R.; Hoj, P. B.; Dry, P. R.; Ford, C.; Iland, P. G. Canopy microclimate and berry composition: the effect of bunch exposure on the phenolic composition of Vitis vinifera L. cv. Shiraz grape berries. Aust. J. Grape Wine Res. 2000, 6, 141-149.

(33) Castellarin, S.; Di Gaspero, G. Transcriptional control of anthocyanin biosynthetic genes in extreme phenotypes for berry pigmentation of naturally occurring grapevines. BMC Plant Biol. 2007, 7, 46.

(34) Bogs, J.; Ebadi, A.; McDavid, D.; Robinson, S. P. Identification of the flavonoid hydroxylases from grapevine and their regulation during fruit development. Plant Physiol. 2006, 140, 279-291.

(35) Castellarin, S. D.; Pfeiffer, A.; Sivilotti, P.; Degan, M.; Peterlunger, E.; Di Gaspero, G. Transcriptional regulation of anthocyanin biosynthesis in ripening fruits of grapevine under seasonal water deficit. Plant Cell Environ. 2007, 30, 1381-1399.

(36) Stafford, H. A. Flavonoid evolution: an enzymic approach. Plant Physiol. 1991, 96, 680-685.

(37) Castellarin, S. D.; Di Gaspero, G.; Marconi, R; Nonis, A.; Peterlunger, E.; Paillard, S.; Adam-Blondon, A. F.; Testolin, R. Colour variation in red grapevines (Vitis vinifera L.): genomic organisation, expression of flavonoid $3^{\prime}$-hydroxylase, flavonoid $3^{\prime}, 5^{\prime}$-hydroxylase genes and related metabolite profiling of red cyanidin-/blue delphinidin-based anthocyanins in berry skin. BMC Genomics 2006, 7, 12.

(38) Downey, M. O.; Harvey, J. S.; Robinson, S. P. The effect of bunch shading on berry development and flavonoid accumulation in Shiraz grapes. Aust. J. Grape Wine Res. 2004, 10, 55-73.

(39) Bindon, K.; Dry, P.; Loveys, B. Influence of partial rootzone drying on the composition and accumulation of anthocyanins in grape berries (Vitis vinifera cv. Cabernet Sauvignon). Aust. J. Grape Wine Res. 2008, 14, 91-103.

(40) Wang, H.; Cao, G.; Prior, R. L. Oxygen radical absorbing capacity of anthocyanins. J. Agric. Food Chem. 1997, 45, 304-309.

(41) Kolb, C. A.; Käser, M. A.; Kopecký, J.; Zotz, G.; Riederer, M.; Pfuindel, E. E. Effects of natural intensities of visible and ultraviolet radiation on epidermal ultraviolet screening and photosynthesis in grape leaves. Plant Physiol. 2001, 127, 863-875. 